\title{
Inputs to Combination-Sensitive Neurons in the Medial Geniculate Body of the Mustached Bat: The Missing Fundamental
}

\author{
Jeffrey J. Wenstrup and Carol D. Grose \\ Department of Neurobiology, Northeastern Ohio Universities College of Medicine, Rootstown, Ohio 44272
}

This study examined projections to combination-sensitive neurons in the medial geniculate body of the mustached bat. These specialized neurons respond to the combination of two temporally and spectrally distinct components of the bat's sonar pulse and echo, encoding target information. Combination-sensitive neurons respond to the bat's sonar fundamental, between 24-31 kHz, in conjunction with a higher harmonic signal. They are thought to be formed in the medial geniculate body (MGB) by convergent input from inferior colliculus representations of $24-31 \mathrm{kHz}$ and higher frequencies. This study used anterograde and retrograde tract-tracing methods in conjunction with physiological recording to test this MGB convergence hypothesis.

In anterograde tracing experiments, multiple deposits of two different tracers were placed in the central nucleus of the inferior colliculus (ICC), one tracer in the 24-31 kHz region and another in an $\mathrm{ICC}$ representation responding to a higher sonar harmonic. We found only limited overlap in the MGB labeling patterns of the two tracers, and little in many areas where combination-sensitive neurons are common. In retrograde tracing experiments, a single deposit of tracer was placed at a combination-sensitive recording site in the MGB. With the deposit mostly limited to combinationsensitive MGB areas, labeling in 24-31 kHz representations of the ICC was absent or minor. These results suggest that many combination-sensitive neurons in the MGB do not receive 24-31 kHz ICC input.

The strongest inputs to combination-sensitive MGB regions originate in high-frequency representations of the ICC and combination-sensitive regions of auditory cortex. Additional projections arrive from the thalamic reticular nucleus, external nucleus of the inferior colliculus, and pericollicular tegmentum. Each projection may contribute to the 24-31 kHz sensitivity of combination-sensitive neurons in the medial geniculate body.

[Key words: medial geniculate body, inferior colliculus, auditory cortex, biosonar, combination sensitive, complex sounds, auditory pathways, bat]

\footnotetext{
Received Oct. 3, 1994; revised Jan. 13, 1995; accepted Jan. 20, 1995.

We thank Z. M. Fuzessery and G. D. Pollak for helpful comments on the manuscript, F.-M. Chen for the software, D. L. Heeter for secretarial assistance, and the Natural Resources Conservation Authority of Jamaica for permission to collect the bats. This work was supported by United States Public Health Service grants to J.J.W. (R29 DC00937, S07 RR05806).

Correspondence should be addressed to Jeffrey J. Wenstrup, Department of Neurobiology, Northeastern Ohio Universities College of Medicine, P.0. Box 95, Rootstown OH 44272-0095.

Copyright (C) 1995 Society for Neuroscience 0270-6474/95/154693-19\$05.00/0
}

Acoustically guided behavior requires the analysis of spectrally and temporally complex signals. The auditory system first analyzes these sounds by means of a spectral decomposition at the inner ear, then transmits the results of this analysis through frequency-specific channels of tonotopically organized central auditory pathways. Further analysis of complex sounds often requires the reverse of this spectral analysis, involving neuronal integration that combines spectrally distinct inputs in the appropriate temporal relationships. Neural systems performing these operations underlie acoustic behaviors including communication (Mudry et al., 1977; Fuzessery and Feng, 1983; Margoliash and Fortune, 1992; Olsen, 1994), sound localization (Takahashi and Konishi, 1986), and biosonar (Suga, 1988; Schuller et al., 1991; Maekawa et al., 1992).

Temporally sensitive, spectrally specific integration is a hallmark of the populations of combination-sensitive neurons in the forebrain of the mustached bat, Pteronotus parnellii (Suga, 1990). These neurons display facilitated responses when two sonar signal components of very different frequency are combined-at the appropriate frequencies, amplitudes, and timing (O'Neill and Suga, 1982; Suga et al., 1983; Taniguchi et al., 1986). Their facilitation requirements suggest that they are designed to respond to the combination of a fundamental component of the bat's outgoing sonar pulse and a higher harmonic component of the returning echo. Some of these neurons are particularly sensitive to the delay between the two components, encoding target distance (O'Neill and Suga, 1982; Suga et al., 1983; Suga and Horikawa, 1986), while others respond more sensitively to the frequencies of the two components, perhaps encoding the relative velocity of the target (Suga et al., 1983; Suga, 1988). Still others may perform different target analyses (Fitzpatrick et al., 1993).

Originally found in cortical areas outside what is believed to be primary auditory cortex, combination-sensitive neurons have now been recorded in primary auditory cortex (Fitzpatrick et al., 1993) and in the medial geniculate body (MGB) (Olsen and Suga, 1991a,b). Since a previous study reported no facilitating combination-sensitive neurons in the inferior colliculus (O'Neill, 1985), it is generally assumed that combination-sensitive responses originate in the MGB. Further, these MGB responses are thought to result from the convergent inputs of inferior collicular neurons representing the fundamental signal and a higher harmonic of sonar or communication signals. We call this the MGB convergence hypothesis.

The present study examined this hypothesis. First, different anterograde tracers were placed in two regions of the central nucleus of the inferior colliculus (ICC), one representing the fundamental sonar component and the second representing some 
higher sonar harmonic. Do the two anterngrade labeling patterns overlap in MGB regions where combination-sensitive neurons occur? Second, a retrograde tracer was placed at a combinationsensitive MGB recording site. Do such tracer deposits label ICC representations of both the fundamental signal and higher sonar harmonics? Finally, we examined inputs to MGB combinationsensitive regions from sources other than the ICC. The results raise doubt that convergent ICC inputs provide the central mechanism by which combination-sensitive neurons are created.

Where this integration occurs, and from what sources, are questions central to understanding the computational mechanisms used by these neurons to analyze complex sounds. For instance, the combination-sensitive neurons that are sensitive to pulse-echo delay seem to depend on delayed excitation to produce a facilitated response. What mechanism(s) creates this delayed excitation? Do these mechanisms rely exclusively on integration of ascending information, or might descending information play a role? To answer these questions, a first step is to identify the site of convergence of frequency-specific information.

Preliminary reports have appeared (Wenstrup, 1992; Wenstrup and Grose, 1993).

\section{Materials and Methods}

Deposits of anterograde and/or retrograde tracers were placed within physiologically defined regions of the ICC or MGB in greater mustached bats (Pteronotus parnellii parnellii). Sixtecn bats, capturcd in Jamaica, West Indies, supply the central data used in this report. Information was also used from an additional 23 animals, part of a larger study of combination-sensitive neurons in the midbrain and thalamus of the mustached bat. All procedures on the animals were approved by the local Institutional Animal Care and Use Committee.

\section{General experimental procedures}

Surgical procedures. The dorsal surface of the inferior colliculus or cerebral cortex was exposed in bats anesthetized with methoxyflurane (Metofane, Pitman-Moore, Inc., Mundelein, IL) in combination with sodium pentobarbital $(5 \mathrm{mg} / \mathrm{kg}$, i.p.; Nembutal, Abbott Laboratories, North Chicago, IL) and acepromazine (2 mg/kg, i.p.; Med-Tech, Inc., Buffalo, NY). A midline incision was made in the skin overlying the skull and the muscles were reflected laterally. A tungsten ground electrode was cemented into the right cerebral or cerebellar cortex, and a small hole (usually less than $0.5 \mathrm{~mm}$ ) was placed in the skull over the appropriate brain region. A metal pin, cemented to the skull and secured to a restraining apparatus, maintained the head in a uniform position during physiological experiments. After application of a long-lasting local anesthetic (lidocaine, Elkins-Sinns, Inc., Cherry Hill, NJ) and a topical antibiotic, the animal was placed in a holding cage to recover from the surgery.

Acoustic stimulation and recording. Usually beginning the day after surgery, physiological recordings were obtained from awake animals placed in a Plexiglas restraining apparatus in a heated and humidified experimental chamber. If the bat struggled or showed signs of discomfort, it was lightly sedated with a cocktail of Nembutal $(5 \mathrm{mg} / \mathrm{kg}$, s.c.) and acepromazine ( $2 \mathrm{mg} / \mathrm{kg}$, s.c.), or removed from the apparatus. Between electrode penetrations, the bat was offered water from a medicine dropper. Recording sessions generally lasted $6-8 \mathrm{hr}$. For any animal, there were no more than three sessions over as many days.

Acoustic stimulation and data acquisition were controlled by an Apple Macintosh IIfx computer running custom-made applications within the LABVIEW environment (National Instruments). Tone or noise bursts (3-30 msec duration, $1 \mathrm{msec}$ rise-fall times, 3-4/sec) were generated by shaping the output of a computer-controlled, multichannel signal generator (Hewlett-Packard model 8904A) with switches (Tucker-Davis Technologies model SW1). The digitally generated sinusoids from the signal generator were accurate to less than $1 \mathrm{~Hz}$. The output of the switch was connected to an attenuator (Tucker-Davis Technologies model PA3), then fed to a power amplifier (Parasound model HCA-800II) and a speaker (Technics leaf tweeter, model EAS 10TH400B) placed $30 \mathrm{~cm}$ away from the bat and $25^{\circ}$ into the sound field contralateral to the recording electrode. In some experiments, two different signals were generated, switched, and attenuated separately. These were added (Tucker-Davis Technologies model ADD1) before being amplified.

The acoustic properties of the entire system were tested using a calibrated microphone (Brüel and Kjaer model 4135) placed in the position normally occupied by the bat's head. There was a smooth, gradual decrease in the sound pressure from $10 \mathrm{kHz}$ to $120 \mathrm{kHz}$ of about $2.7 \mathrm{~dB}$ per $10 \mathrm{kHz}$. Distortion components in the speaker output were at least $60 \mathrm{~dB}$ below the sound pressure level (SPL) of the signal, as measured by a LABVIEW application performing a fast Fourier transform of the digitized (1 $\mathrm{MHz}$ sampling rate, National Instruments model NBA2000) microphone signal.

The evoked activity of single units and multiunit clusters was recorded with micropipettes having tip diameters of 5-10 $\mu \mathrm{m}$ (resistances of $1-15$ Megohms) and filled with one of several tracers: wheat germ agglutinin-conjugated to horseradish peroxidase (WGA-HRP, Sigma Chemical Co., St. Louis, MO), biocytin (Sigma Chemical Co.), or cholera toxin B-subunit (CTb, List Biologicals, Campbell, CA). Tracer-specific techniques are described in a later section. Electrodes were advanced by a hydraulic micropositioner (David Kopf Instruments, model 650). Extracellular action potentials wcre amplificd, filtered (bandpass, $500-6000 \mathrm{~Hz}$ ), and sent through a window discriminator (Frederick Haer and Co. model 74-60-3). The pulse output of the window discriminator was digitized at $10 \mathrm{kHz}$ (National Instruments, Inc., model NBMIO-16X). Peristimulus time (PST) histograms, raster displays, and statistics on the neural responses were generated by the LABVIEW application. Multiunit responses that were analyzed consisted of stimuluslocked clusters of clearly defined spikes; the threshold of the window discriminator was adjusted to detect spike activity exceeding the baseline noise level. The window discriminator output was also displayed audiovisually.

Using surface landmarks or a stereotaxic procedure as a guide, electrodes were placed to record neural activity within the ICC or MGB. Responses were usually sampled at $100-200 \mu \mathrm{m}$ intervals. Response properties were evaluated using tone or noise bursts. The best frequency (the frequency requiring the lowest intensity to elicit stimulus-locked spikes) and threshold at best frequency (defined as the lowest intensity required to elicit one or more spikes to each of five consecutive stimuli) were measured at each recording site. In most cases, the Q10dB measure of tuning sharpness was obtained at the deposit site, since very sharp tuning distinguishes ICC and MGB neurons analyzing some components of the biosonar signal (Pollak and Bodenhamer, 1981; O'Neill, 1985; Olsen and Suga, 1991a).

In the MGB, where many combination-sensitive neurons occur (OIsen and Suga, 1991a,b), we tested neurons with an array of signals combining frequencies in the fundamental sonar component with frequencies in a higher harmonic sonar component. If a combination-sensitive response was obtained, measures of frequency- and/or delay-dependent facilitation (or inhibition) were obtained. Our goal was to clearly establish that neurons at deposit sites were combination sensitive, not to describe these neurons in all their physiological details. Thus, we used only tone burst stimuli, to which nearly all combination-sensitive MGB neurons respond (Olsen and Suga, 1991a,b). ICC neurons in this study were not tested for combination-sensitive responses.

At the end of a penetration, response properties were again characterized in the region to receive the tracer deposit. Iontophoretic deposits were made using a constant current source (Midgard model CS4). $\Lambda \mathrm{fter}$ the deposit, the electrode was kept in position for $5 \mathrm{~min}$, then removed from the brain. On the following day, physiological recordings and tracer deposits were obtained from another region of the same structure, or from the same structure on the other side of the brain. For experiments in which two different tracers were placed in the ICC, WGA-HRP deposits were placed on $1 \mathrm{~d}$, biocytin deposits placed on the next, and the animal perfused on the third day. In retrograde tracing experiments, WGA-HRP or CTb deposits were placed in each MGB on consecutive days, and the animal perfused $1 \mathrm{~d}$ (for WGA-HRP experiments) or $3 \mathrm{~d}$ (for CTb experiments) following the second deposit.

Histology. After tracer deposits, the bat was returned to its holding cage. The animal was perfused after having been deeply anesthetized with Nembutal $(60 \mathrm{mg} / \mathrm{kg}$, i.p.). When nociceptive reflexes were eliminated, the chest cavity was opened and the animal was perfused through the heart with phosphate-buffered saline and an aldehyde fixative. After the brain case was opened, the brain was blocked in a consistent plane (the plane of most electrode penetrations), inclined about $15^{\circ}$ from dorsal and caudal to ventral and rostral. This plane is 
A

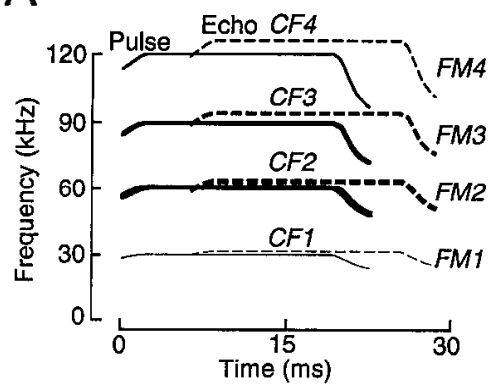

Biosonar Frequency Bands

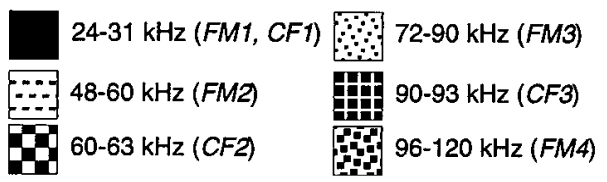

B

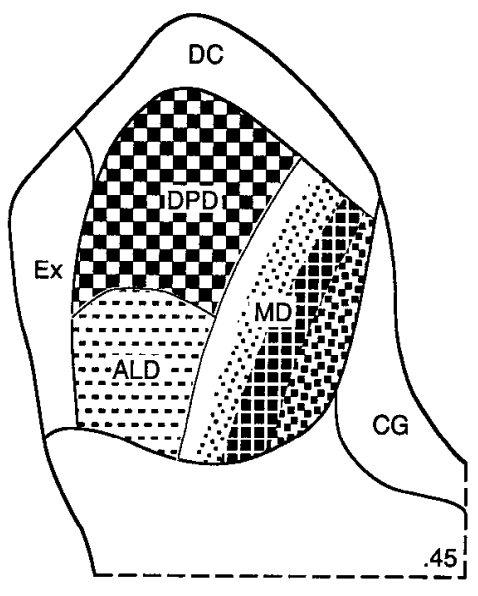

C

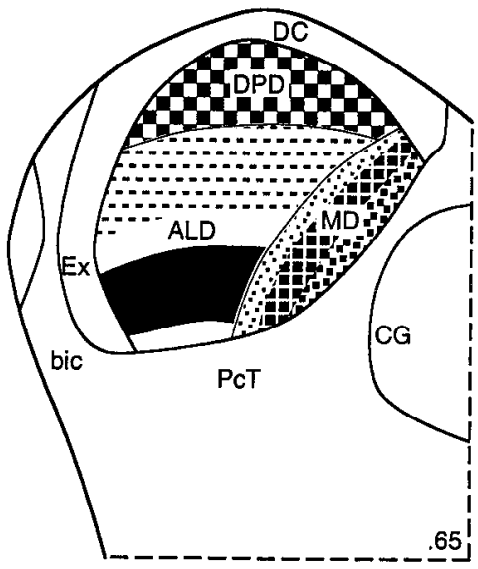

Figure 1. A, Sound spectrogram of mustached bat sonar pulse and echo. The echo is delayed and (Doppler) shifted upward in frequency. Lettering indicates the individual components of four sonar harmonics: constant frequency $(C F I-4)$ and frequency modulated $(F M I-4)$. $B$ and $C$, Schematic illustration of the representation of biosonar signal components within the tonotopic organization of the central nucleus of the inferior colliculus (ICC). Numbers at lower right of ICC sections indicate location along the caudal-to-rostral dimension of the inferior colliculus; the section in $B$ is more caudal than the section in $C$.

consistent with prcvious anatomical and physiological studies of the inferior colliculus and MGB (Wenstrup et al., 1986; Wenstrup et al, 1988; Wenstrup et al., 1994). Each brain was refrigerated overnight in a $30 \%$ sucrose-phosphate buffer solution before sectioning. Brains were sectioned transversely on a freezing microtome at a thickness of $30 \mu \mathrm{m}$. All sections from the cochlear nucleus through the auditory cortex were collected into cold $0.1 \mathrm{M}$ phosphate buffer or phosphate-buffered saline. Every third section was processed by a different protocol; one of the three series was stained with cresyl violet.

\section{Tracer-specific techniques}

WGA-HRP. Electrodes were filled with either $2 \%$ WGA-HRP in $0.9 \%$ $\mathrm{NaCl}$ or $4 \%$ WGA-HRP in $0.9 \mathrm{M} \mathrm{NaCl}, 0.1 \mathrm{M}$ phosphate buffer $(\mathrm{pH}$ adjusted to 7.6). For iontophoresis, a pulsed current ( +1 p.A, 7 sec ON/7 sec OFF) was applied for 3.5-20 min, depending on the desired size of the deposit and the ionic concentration of the electrode solution. Animals were perfused $24-18 \mathrm{hr}$ after WGA HRP deposits, using a mixed aldehyde fixative $(1.25 \%$ glutaraldehyde and $1 \%$ paraformaldehyde (Mesulam, 1982), or $0.5 \%$ glutaraldehyde and $2 \%$ paraformaldehyde), followed by a cold $\left(4^{\circ} \mathrm{C}\right)$ solution of $10 \%$ sucrose in phosplate buffer. Both tetramethylbenzidine (TMB) and diaminobenzidine (DAB) were used as chromogens in different series. One TMB series was counterstained with neutral red (Mesulam, 1982), while the DAB series was counterstained with cresyl violet.

Biocytin. Procedures using biocytin, described previously (Wenstrup et al., 1994), were adapted from King et al. (1989) and Izzo (1991). Electrodes were filled with 5\% biocytin (Sigma Chemical Co.) and 0.9 $\mathrm{M} \mathrm{NaCl}, 0.1$ M phosphate buffer, $\mathrm{pH}$ adjusted to 7.6. Biocytin was iontophoresed $(+5.0 \mu \mathrm{A})$ using either continuous current (10 min duration) or pulsed current ( $20 \mathrm{~min}, 7 \mathrm{sec} \mathrm{ON} / 7 \mathrm{sec} \mathrm{OFF}$ ). Animals were perfused $20-26 \mathrm{hr}$ after biocytin deposits, using a mixed aldehyde fixative $(0.5 \%$ glutaraldehyde and $2 \%$ paraformaldehyde). Since biocytin experiments were usually combined with WGA-HRP experiments, only one of the three series was processed to visualize biocytin. In this series, sections were first processed with $\mathrm{DAB}$ (brown reaction product) to visualize WGA-HRP deposit sites, then biocytin labeling was shown using an avidin-biotin-peroxidase procedure (Vector Laboratories, Burlingame, $\mathrm{CA}$ ) and a heavy metal intensification of the DAB reaction (black reaction product; Adams, 1981). This series was counterstained with cresyl violet.

Cholera toxin, $B$-subunit $(C T b)$. CTb procedures were adapted from Luppi et al. (1988). Electrodes were filled with $1 \% \mathrm{CTb}$ (List Biologicals) in phosphate-buffered saline $(\mathrm{pH} 7.4)$. Tracer deposits were made with a pulsed current $(+2 \mu \mathrm{A}, 7 \mathrm{sec} \mathrm{ON} / 7 \mathrm{sec} \mathrm{OFF})$ for $10 \mathrm{~min}$. Animals were perfused $3-5$ d later with a $4 \%$ paraformaldehyde fixative. Tracer was visualized using immunohistochemistry and an avidin-bio- tin-peroxidase procedure (Vector Laboratories). After blocking in $3 \%$ normal rabbit serum plus $0.2 \%$ Triton $\mathrm{X}$, free-floating sections were incubated in goat anti-CTb (1:40,000, List Biologicals) for $62-70 \mathrm{hr}$ at $4^{\circ} \mathrm{C}$. Heavy metal-intensified DAB was used as the chromogen.

\section{Data analysis}

To show deposits, we illustrate both a central core of dense reaction product and a surrounding zone, containing heavily labeled perikarya, which may be a better estimate of the effective uptake zone for the tracer. Damage to axons of passage was unusual in WGA-HRP deposits, but sometimes occurred in biocytin experiments. To minimize errors of interpretation in two-tracer experiments, we specifically placed biocytin in the regions that did not contain axons originating at the site of WGAHRP deposits, and we studied the location of apparent damaged axons or filled somata. In the text, filled cells away from the injection site are noted.

$\Lambda$ terograde and retrograde labeling based on WGA-HRP transport was plotted from TMB-processed sections using a drawing tube and dark-field illumination (234X, N.A. 0.4, plan apochromat). Anterograde and retrograde labeling based on biocytin or $\mathrm{CTb}$ was plotted using bright-field illumination ( $468 \times$, N.A. 0.7 , plan apochromat). The dots used to display anterograde WGA-HRP transport represent both terminal and preterminal labeling, while those showing biocytin labeling represent presumptive terminals. Counts of retrogradely labeled cells in WGA-HRP or CTb experiments were made from every section in one series, i.e., from every third section.

All plots were scanned at 300 dots per inch and imported into a graphics application (CANVAS, Deneha Software), where subdivisions, drawn and scanned separately, were added. To improve visualization, the fine dots used to plot anterograde labeling were slightly coarsened in an image-processing application (РНОтовIIOP, $\Lambda$ dobe, Inc.) before importation into CANVAS. In experiments where the overlap between WGA-HRP and biocytin transport was of interest, labeling patterns from adjacent sections were superimposed in CANVAS, using blood vessels and tissue outlines as guides. If necessary, computer images of DABprocessed sections were reduced in scale to compensate for the greater shrinkage due to TMB processing. In the histological material from each experiment, cytoarchitectonic boundaries were drawn from Nisslstained sections, based on previous work in the inferior colliculus (Zook and Casseday, 1982; Zook et al., 1985) and MGB (Winer and Wenstrup, 1994a,b).

\section{Results}

An echolocating mustached bat analyzes complex acoustic signals consisting of a sonar pulse and one or more delayed echoes 
(Fig. 1A). The fundamental signal includes a constant frequency segment $(\mathrm{CF} 1, \sim 30 \mathrm{kHz})$ followed by a frequency modulated sweep (FM1, 30-24 kHz), with several higher harmonics: CF2 $(\sim 60 \mathrm{kHz}), \mathrm{CF} 3(\sim 90 \mathrm{kHz}), \mathrm{CF} 4(\sim 120 \mathrm{kHz})$, FM2 $(60-48$ $\mathrm{kHz}$ ), FM3 (90-72 kHz), and FM4 (120-96 kHz) (Novick, 1963). These values are approximate because individual bats use slightly different frequencies, because Doppler shifts elevate echo frequencies, and because the bats compensate for Doppler shifts by lowering frequencies in emitted pulses (Schnitzler, 1970; Henson et al., 1982). In the ICC, biosonar signals and other sounds are analyzed within a tonotopic representation that fills three architectonically distinct divisions (Fig. $1 B, C$ ): the anterolateral $(10-59 \mathrm{kHz})$, the dorsoposterior $(60-63 \mathrm{kHz})$, and the medial (64-120 kHz) (Zook et al., 1985; O'Neill et al., 1989; Wenstrup et al., 1994).

Combination-sensitive neurons in the medial geniculate body (MGB) respond to frequencies within the FM1 or C.F1 components in conjunction with a higher harmonic component. Classes of combination-sensitive neurons, named for the sonar components to which they normally respond, include CF1-CFn (where $n=2$ or 3 ), FM1-FM $n(n=2,3$, or 4$)$, and FM1-CF2. These are common in the rostral half of the MGB, including the dorsal and rostral pole nuclei and the medial part of the ventral division (Olsen and Suga, 1991a,b; this study).

\section{Part 1: projections of ICC representations to the $M G B$}

If ICC neurons from CFl or FM1 representations converge with those from higher harmonic representations to form combination-sensitive neurons in the MGB, then sensitive anterograde tracing experiments should obtain two results. First, sizable tracer deposits in the ICC representation of the fundamental frequencies $(24-31 \mathrm{kHz}$ ) should label extensive areas in the rostral half of the MGB where combination-sensitive neurons are common. Second, this pattern of labeling should overlap with other labeling obtained from high-frequency ICC tracer deposits, and this overlap should occur in the combination-sensitive regions of the MGB. The experiments below used two anterograde tracers to test these predictions.

Projections of $\mathrm{CF} 1$ and $\mathrm{CF} 2$ representations in ICC. Projections that may contribute to CF1-CF2 or FM1-CF2 neurons in the MGB were examined in an experiment shown in Figure 2. Anterograde MGB labeling was examined after three WGAHRP deposits in regions of the ICC tuned near $30 \mathrm{kHz}$, and seven deposits of biocytin in ICC regions tuned near $60 \mathrm{kHz}$ (Fig. $2 G-J$ ). This case was of high quality, with restricted deposit sites and strong, focal, anterograde labeling.

The WGA-HRP deposit zone was limited to the rostral part of the anterolateral division. It probably included frequency representations from $20 \mathrm{kHz}$ to $40 \mathrm{kHz}$, thus labeling both $\mathrm{CF} 1$ and FM1 outputs. The deposit zone did not spread to the caudal part of the anterolateral division, the dorsoposterior division, or the medial division. Thus, it did not include ICC regions analyzing the second, third, or fourth sonar harmonics, from $48-120 \mathrm{kHz}$.

In the MGB, most WGA-HRP labeling was restricted to nuclei along the perimeter of the MGB (Fig. $2 A-F$ ). Substantial labeling was observed in the lateral part of the ventral division (V1), the part of the tonotopically organized ventral division representing 10-59 $\mathrm{kHz}$. Other labeling occurred in the dorsal nucleus (D), the suprageniculate nucleus $(\mathrm{Sg})$, the medial division (M), and the rostral pole nucleus (RP) in its ventral, medial, and rostral extreme. Very little labeling was in the medial part of the ventral division $(\mathrm{Vm})$, the tonotopic representation of $60-120$
$\mathrm{kHz}$, or the central part of the rostral pole nucleus. This pattern is nearly identical to one observed following deposits of other tracers at ICC sites tuned to $25 \mathrm{kHz}$ (Wenstrup et al., 1994) and is characteristic of experiments in which tracer deposits are limited to the rostral part of the anterolateral division. Whether placed in 25 or $30 \mathrm{kHz}$ representations, these deposits probably labeled ICC efferent fibers from both the population responding to the FM1 sonar component and the population responding to the CF1 sonar component.

Biocytin deposits filled much of the dorsoposterior division, the ICC representation of $60-63 \mathrm{kHz}$, which analyzes the CF2 sonar component (Fig. 2G,H). Some tracer extended beyond the ICC into the external nucleus and the dorsal cortex. A few faintly labeled cells in the medial division and the caudal part of the anterolateral division indicated that a small amount of anterograde labeling may originate from $48-59 \mathrm{kHz}$ or $64-120 \mathrm{kHz}$ ICC neurons. The $60 \mathrm{kHz}$ responses at deposit sites were very sharply tuned, with Q10dB values of $133-400$. Such sharply tuned responses are characteristic of neurons in the dorsoposterior division of the ICC (Pollak and Bodenhamer, 1981; O'Neill, 1985). MGB labeling after these deposits was heaviest in $\mathrm{Vm}$, particularly the dorsal and lateral parts, and in the dorsal nucleus (Fig. $2 A-C$ ). Other regions were lightly labeled, including the suprageniculate nucleus, the medial division, and $\mathrm{Vl}$. Although the labeling in Vl is puzzling, we have routinely observed patchy, light labeling here after higher frequency deposits in the ICC. These results agree well with previous results using horseradish peroxidase as the tracer (Wenstrup et al., 1994).

There were several regions where some degree of overlapping labeling occurred (Fig. 2, encircled areas). These were most apparent in Vl (Fig. $2 B-D$ ), the suprageniculate nucleus (Fig. $2 A$ ), the medial division (Fig. $2 A$ ), and the dorsal nucleus (Fig. $2 B, C$ ), a region where combination-sensitive neurons occur (Olsen and Suga, 1991a; this study). This limited overlap suggests that some 24-31 kHz (FM1 and CF1) and CF2 projections from the ICC may converge to form combination-sensitive MGB neurons. However, this experiment did not reveal evidence of the widespread 24-31 kHz ICC input that we expected of the MGB convergence hypothesis. In fact, a striking aspect of these results was the absence of $24-31 \mathrm{kHz}$ labeling in $\mathrm{Vm}$ and the rostral pole nucleus throughout much of the rostral half of the MGB (Fig. $2 C-F$ ), even though many of the combination-sensitive neurons we recorded were located there.

Projections of $24-31 \mathrm{kHz}$ and FM3 representations in ICC. The restricted size of the deposit sites in the previous experiment (Fig. 2) may have limited overlapping label in the MGB. In particular, tracer deposits near $30 \mathrm{kHz}$ may not have included the entire ICC representation of $\mathrm{CF} 1$ frequencies. By contrast, Figure 3 illustrates an experiment in which WGA-HRP deposits filled nearly all of the $24-31 \mathrm{kHz}$ representation in the ICC.

Three deposits were placed in the rostral part of the anterolateral division, at sites tuned to $27.9-31.1 \mathrm{kHz}$ (Fig. 3I,J). Due to the size of the deposits and the leakage of tracer from the pipette, the deposit zone included the caudal part of the anterolateral division and some of the dorsoposterior division (Fig. $3 H$ ). Effectively, this deposit probably included at least parts of ICC representations from $10-63 \mathrm{kHz}$. As confirmation that this deposit zone extended into $48-59 \mathrm{kHz}$ and $60-63 \mathrm{kHz}$ ICC representations, many retrogradely labeled cells were observed in the caudal part of the anterolateral division and the dorsoposterior division of the contralateral ICC. However, the deposit did not extend into the medial division, which represents frequencies 

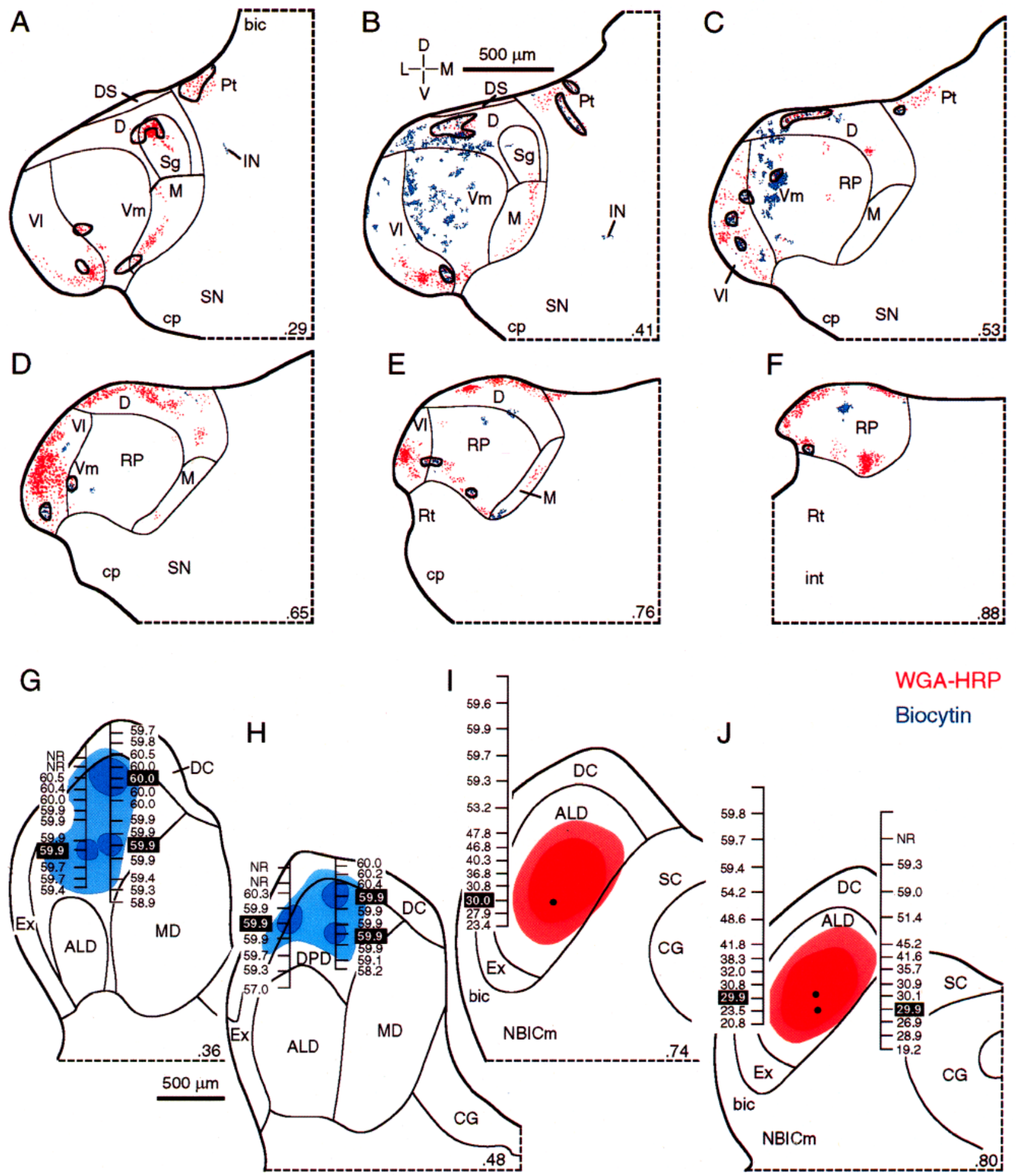

Figure 2. Results of an experiment that placed tracer deposits in $30 \mathrm{kHz}(\mathrm{CF} 1)$ and $60 \mathrm{kHz}(\mathrm{CF} 2)$ representations of the ICC. A-F, Distribution in the MGB of anterograde WGA-HRP labeling (in red) and biocytin labeling (in blue). Overlap of WGA-HRP and biocytin labeling in the MGB was limited to encircled regions (thicker lines). Large areas of the rostral pole, where combination-sensitive neurons occur, are unlabeled by the WGA-HRP deposits. $G-J$, Best frequencies and tracer deposit sites in penetrations through the ICC. Black dots show centers of WGA-HRP deposits. Solid colored region shows most heavily labeled part of deposit site, while the shaded region indicates our estimate of the zone of transport. Whiteon-black numbers show best frequencies at deposit sites. Penetrations in $G$ and $H$ lay in the plane of section, while those in $I$ and $J$ crossed the plane of section. Numbers at lower left of sections show the caudal-to-rostral position of the section through the MGB or IC. 

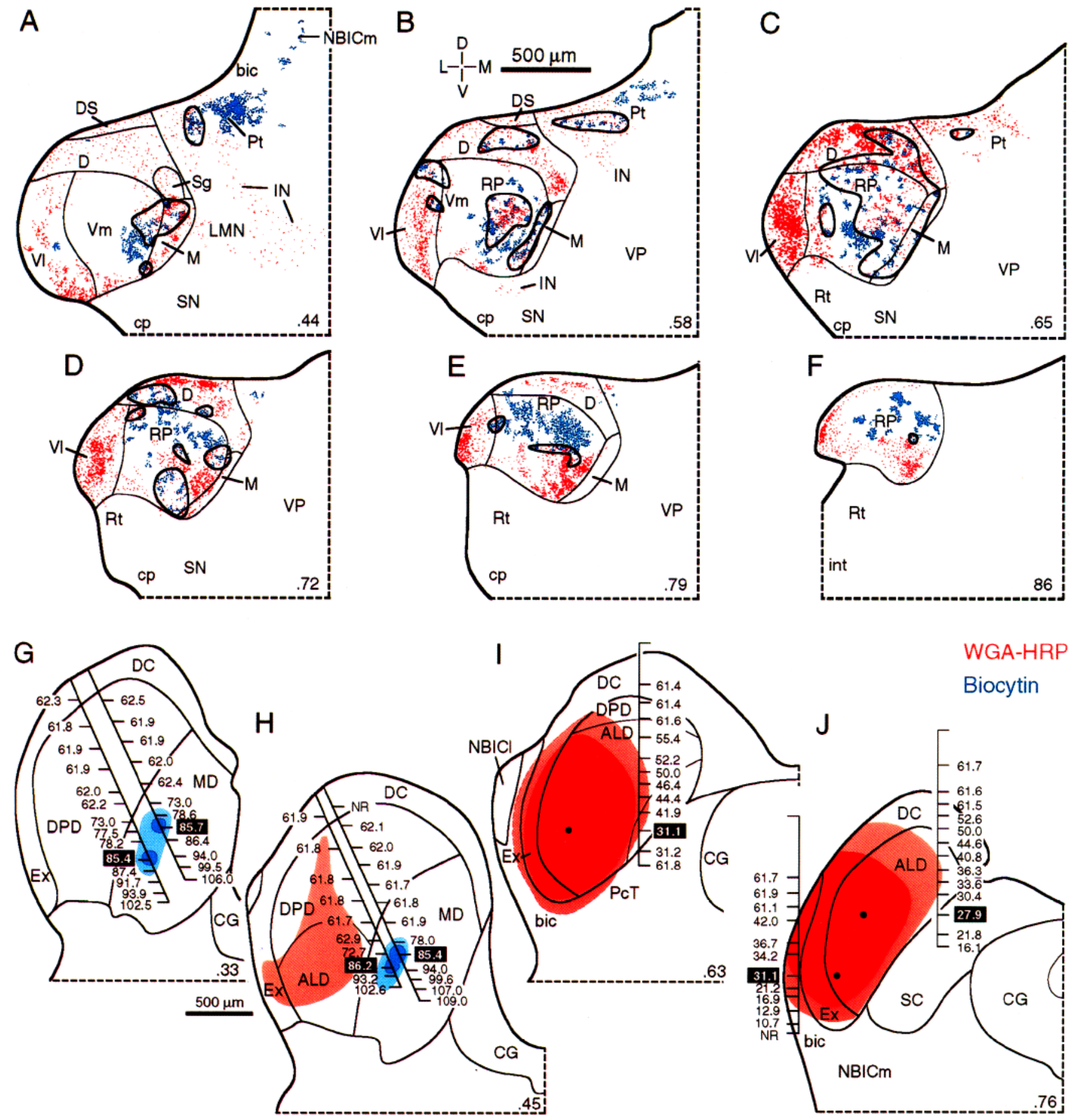

Figure 3. Results of an experiment that placed deposits in 28-31 kHz (FM1 and CF1) and 85-86 (FM3) representations of the ICC. WGA-HRP deposits and anterograde labeling were very extensive in this experiment. $A-F$, Distribution in the MGB of anterograde WGA-HRP labeling (in red) and biocytin labeling (in blue). Note the widespread distribution of WGA-HRP labeling, probably the result of the very large deposit zone. Overlap (encircled regions) was extensive, but was nevertheless absent from parts of the rostral pole $(D-F)$ that was labeled by $85-86 \mathrm{kHz}$ biocytin deposits and is combination sensitive. $G-J$, Best frequencies and tracer deposit sites in electrode penetrations through the ICC. The WGA-HRP deposit zone included nearly all of the rostral part of the anterolateral division $(A L D)$ and extended into the caudal ALD, the dorsoposterior division $(D P D)$, and the external nucleus $(E x)$. See Figure 2 legend for protocol.

above $64 \mathrm{kHz}$. The deposit zone also included pericentral nuclei, particularly the external nucleus.

As expected, MGB labeling after these WGA-HRP deposits was widespread (Fig. $3 A-F$ ). All the regions labeled by the more restricted $30 \mathrm{kHz}$ deposits shown in Figure 2 were heavily la- beled here. These included Vl, the suprageniculate nucleus, the dorsal nucleus, the medial division, and the ventromedial part of the rostral pole. Additional labeling occurred in $\mathrm{Vm}$ and the rostral pole nucleus. Much of the rostral pole labeling $(B-E)$ corresponded well to $48-59 \mathrm{kHz}$ label in other experiments 

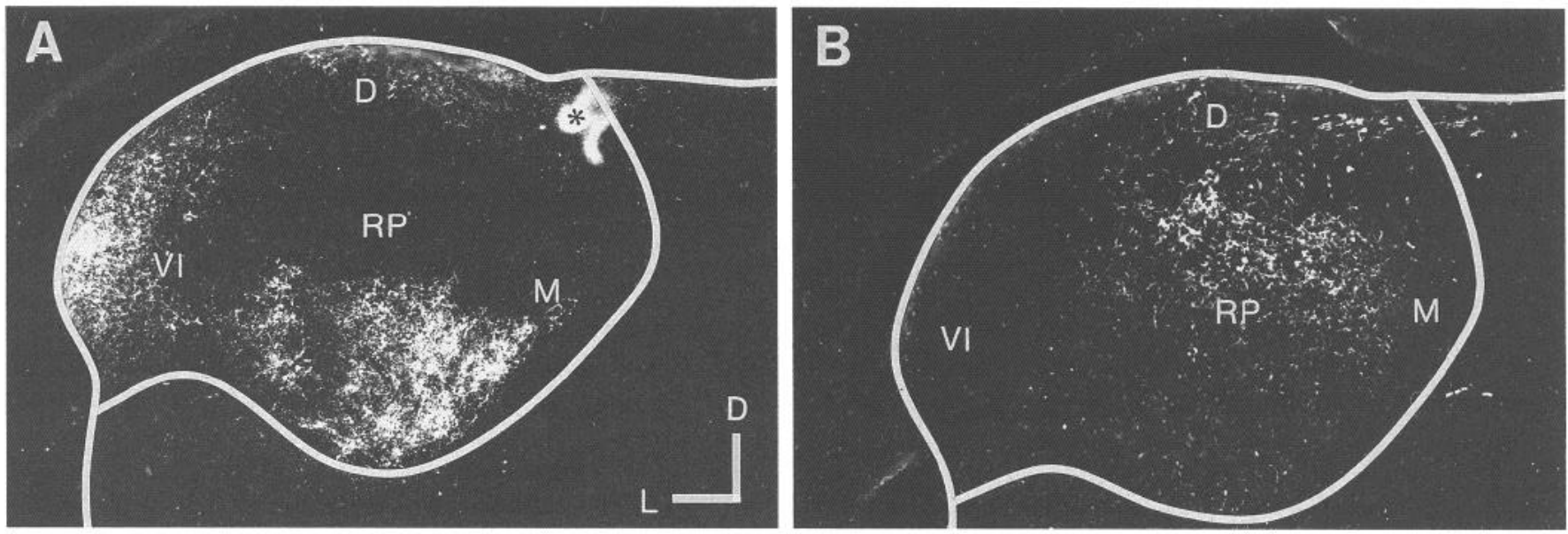

Figure 4. Dark-field photomicrographs of adjacent sections in experiment illustrated in Figure 3, showing WGA-HRP $(A)$ and biocytin $(B)$ labeling. WGA-HRP labeling from very large $28-31 \mathrm{kHz}$ deposits in ICC is intense in the lateral part of the ventral division ( $V l$ ), the ventral part of the rostral pole nucleus $(R P)$, and the medial division $(M)$. Biocytin labeling from $85-86 \mathrm{kHz}$ deposits is heavy in the dorsal part of RP. No WGAHRP labeling occurs in the region of intense biocytin label. Asterisk in A marks artifact. Scale bar (orientation lines), $100 \mu \mathrm{m}$.

(Wenstrup et al, 1994). Lighter label was also observed in the pretectum (Pt) and intralaminar nuclei (IN). The latter probably resulted from the spread of tracer to regions surrounding the ICC, as in the rat (LeDoux et al., 1985). However, despite the extensive anterograde labeling in the MGB, parts of $\mathrm{Vm}$ and the rostral pole nucleus were not labeled (Figs. $3 A-F, 4$ ).

Four biocytin deposits were placed in the medial division of the ICC, at sites tuned to $85.4-86.2 \mathrm{kHz}$ (Fig. $3 G, H$ ). Biocytinfilled cells around these sites were probably restricted to ICC representations in the range $75-94 \mathrm{kHz}$, i.e., the FM3 and CF3 biosonar representations. Physiologically, the deposit site responses were characterized by broad tuning (Q10dB values 8.715.3) and short latency (5-10 msec). After these deposits, there was moderate or heavy MGB labeling in Vm, the dorsal nucleus, the medial division, and several loci in the rostral pole nucleus, especially in the dorsal part (Fig. $3 A-F$ ). The pretectum was heavily labeled (Fig. $3 A$ ), a characteristic feature of projections from FM2, FM3, and FM4 representations in ICC. This labeling pattern agrees well with that reported previously for the 80-85 $\mathrm{kHz}$ representation (Wenstrup et al., 1994).

Overlap in the WGA-HRP and biocytin labeling occurred in several places; the rostral pole nucleus, the dorsal nucleus, the medial division, and the pretectum (Fig. $3 A-C$, encircled areas) are examples. The meaning of this overlap is unclear, since the WGA-HRP deposits were so extensive. Overlap in the dorsal division (Fig. $3 B-D$ ) may represent convergent FM1 and FM3 inputs, since other experiments have separately shown 24-31 $\mathrm{kHz}$ and FM3 inputs (Fig. 2; Wenstrup et al., 1994). In the rostral pole, however, we suggest that much of the overlap (Fig. $3 B-F$ ) is the result of the inadvertent WGA-HRP deposit in the $48-59 \mathrm{kHz}$ ICC representation. This ICC representation is known to project to regions of the rostral pole adjoining and intermixed with those regions receiving $72-90 \mathrm{kHz}$ ICC input (Wenstrup et al., 1994).

The most striking results concerned regions where overlap did not occur. Specifically, parts of the rostral pole nucleus received strong $85-86 \mathrm{kHz}$ input but were virtually unlabeled by the massive WGA-HRP deposits in the anterolateral division of the ICC (Figs. 3D-F, 4). This region contains combination-sensitive neurons (Olsen and Suga, 1991a; see also Figs. 8, 9). WGA-HRP deposits also failed to label other parts of $\mathrm{Vm}$ and the rostral pole nucleus that receive input from other high-frequency ICC representations (Fig. 2; Wenstrup et al., 1994). Some of these regions probably contain combination-sensitive neurons (see Fig. 6).

These anterograde tracer experiments did not reveal evidence of widespread FM1 or CF1 input to some of the regions of the rostral MGB containing combination-sensitive neurons. In the case with more restricted WGA-HRP deposits (Fig. 2), much of the combination-sensitive, rostral MGB was virtually unlabeled. Even with the most extensive WGA-HRP deposit zone (Fig. $3 H-J$ ), we found regions of the rostral MGB that received strong FM3 input, are probably combination sensitive, but are not even weakly labeled by the massive CF1 and FM1 deposits (Figs. $3 D-F, 4)$. These data question whether low-frequency representations of the ICC are a major source of FM1 or CF1 input to some combination-sensitive MGB regions.

Location of the $24-31 \mathrm{kHz}$ representation in ICC. The physiologically guided tracer deposits in the ICC allowed us to establish the locations of $24-31 \mathrm{kHz}$ (FM1 and CF1) representations, information that is used to interpret experiments in the next section. Previous studies showed that frequencies in the $\sim 10-59 \mathrm{kHz}$ range are represented in the anterolateral division (Zook et al., 1985; O'Neill et al., 1989; Wenstrup et al., 1994). Here, anatomically defined fibrodendritic laminae (Zook et al., 1985 ) and physiologically defined frequency-band laminae (O'Neill et al., 1989) extend dorsoventrally and mediolaterally, their edges curving rostrally. There is an orderly progression from the highest frequencies located more caudally $(59 \mathrm{kHz})$ to lower frequencies represented more rostrally. The enlarged 48 $59 \mathrm{kHz}$ representation (FM2) occupies much of the caudal part of the anterolateral division, while the $24-31 \mathrm{kHz}$ frequency band is represented in the rostral part.

Our observations agree closely with the previous reports and supply detailed information that applies to the plane of section used in all experiments of the present study. We have recorded over 1100 single- and multiple-unit responses from 85 anatomically marked penetrations through the inferior colliculus (IC). In the 59 penetrations with deposits placed in the caudal $62 \%$ of the IC, including 22 penetrations passing through the caudal part of the anterolateral division, we did not record any neurons having a best frequency in the range $24-31 \mathrm{kHz}$ (e.g., Figs. 


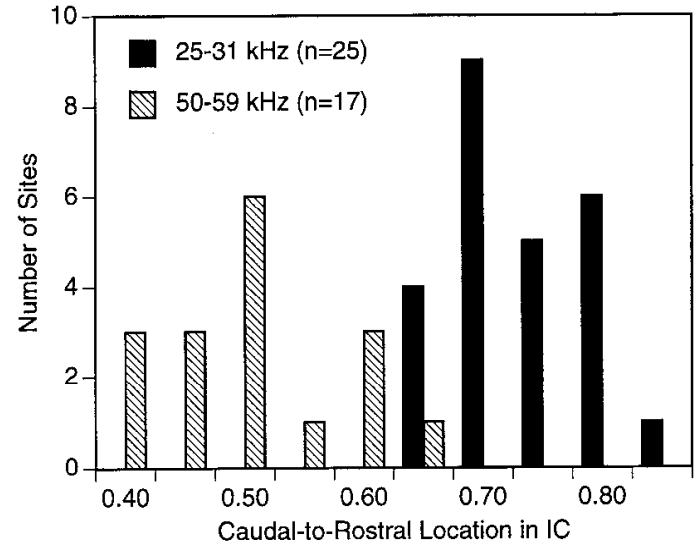

Figure 5. Distribution of ICC deposit sites from frequency bands representing FM1 and $\mathrm{CF} 1(24-31 \mathrm{kHz})$ versus FM2 $(48-59 \mathrm{kHz})$ sonar components, expressed as a fraction of the caudal to rostral dimension of the inferior colliculus $(I C)$. Best frequencies in the $24-31 \mathrm{kHz}$ band were observed only in the rostral IC, from 0.63 to 0.83 along the caudalto-rostral dimension.

$2 G, H ; 3 G, H)$. Some penetrations through the anterolateral division, with deposits as far rostral as $67 \%$ through the caudalto-rostral IC dimension, also revealed no evidence of $2431 \mathrm{kHz}$ best frequency responses.

Despite our attempts to find FM1 and CF1 tonotopic representations and deposit sites more caudally, all $24-31 \mathrm{kHz}$ recording sites and tracer deposit sites were placed in the rostral $38 \%$ of the IC (Fig. 5). Neurons with best frequencies in the FM1 frequency range $(24-29 \mathrm{kHz})$ began somewhat more rostrally than CF1 neurons $(29-31 \mathrm{kHz}$ ); they were recorded only in the rostral $32 \%$ of the IC. By comparison, $48-59 \mathrm{kHz}$ deposit sites were placed caudally in the anterolateral division (Fig. 5). When the two frequency-band representations overlapped in caudal-to-rostral extent, $48-59 \mathrm{kHz}$ neurons were located dorsal to $24-31 \mathrm{kHz}$ neurons (Figs. $2 I, J ; 3 I, J$ ). Thus, for the plane of section used in this study, we consider that FM1 and CF1 tonotopic representations lay exclusively within the rostral part of the anterolateral division, a noncytoarchitectonic region we defined as that part of the anterolateral division located within the rostral $40 \%$ of the IC.

\section{Part 2: ICC inputs to combination-sensitive areas of $M G B$}

Combination-sensitive neurons were abundant in the rostral half of the $\mathrm{MGB}$, the region examined in this study. In this group we include facilitatory FM-FM (see Figs. 7, 8) and CF-CF neurons described previously in MGB (Olsen and Suga, 1991a,b), as well as facilitatory FM1-CF2 neurons (Fig. $6 H$ ) like those recently described in the auditory cortex (Fitzpatrick et al., 1993). We also included two other groups. One was characterized by an excitatory response to high-frequency tones that was inhibited by sounds in the $24-31 \mathrm{kHz}$ range (see Fig. $9 H$ ). Still others displayed excitatory responses to both low- and highfrequency signals, but showed no interaction between the two. The feature common to these neurons was a sensitivity to sounds in the $24-31 \mathrm{kHz}$ range. Each category was recorded in both single- and multiple-unit responses.

Although we did not exhaustively survey the distribution of combination-sensitive neurons throughout the rostral MGB, we observed that these neurons regularly occurred in a few nuclei. Among 22 anatomically marked penetrations through the rostral half of the MGB, nearly all combination-sensitive neurons were in the dorsal nucleus, the rostral pole nucleus, and $\mathrm{Vm}$ (e.g., Figs. $6 G-9 G, 11 B$ ). A few appeared to be in the superficial dorsal nucleus, while none were recorded in $\mathrm{Vl}$ or the medial division.

If the MGB convergence hypothesis is valid, retrograde tracers placed in the combination-sensitive areas of the MGB should label significantly at least two regions of the ICC: one corresponding to the high-frequency responses at the deposit site, and the other within the CF1 or FM1 representations located in the rostral $40 \%$ of the IC. Below are experiments in which we recorded combination-sensitive responses in the MGB, then deposited a retrograde tracer to label ICC and other inputs. In the first, a deposit was placed in a combination-sensitive $\mathrm{CF}_{2}$ region. Next, three experiments with deposits in FM-FM regions are described.

ICC inputs to a combination-sensitive CF2 region of $M G B$. We placed a series of WGA-HRP deposits at CF2 $(60-61 \mathrm{kHz})$ combination-sensitive recording sites in Vm (Fig. 6G). Throughout the electrode penetration of the dorsal nucleus and $\mathrm{Vm}$, most single- and multiple-unit responses were combination sensitive, and all of these responded to the $\mathrm{CF} 2$ component. $\mathrm{CF} 2$ responses in this region are consistent with anterograde tracing studies that showed labeled axon terminals after biocytin deposits in the CF2 representation of the ICC (Fig. 2C). At the center of the deposit zone, the best frequency of facilitation for the fundamental sonar component was $27.5 \mathrm{kHz}$, and the maximum facilitation occurred when the two signals were presented simultaneously (Fig. $6 H$ ). We classified these neurons as FM1-CF2, since their tuning to the low-frequency component was consistent with cortical FM1-CF2 neurons (Fitzpatrick et al., 1993).

Retrograde labeling in the ICC was remarkable for being restricted to a few well-defined regions (Fig. $6 A-F$, Table 1). Most labeled cells $(374,68 \%$ of ICC labeling) were in the dorsoposterior division, where sharply tuned $60-63 \mathrm{kHz}$ responses are found (Fig. 6A-C). There was also a well-defined strip of labeling in the medial division of the ICC. This labeling (Fig. $6 A, B)$ was probably the result of uptake by axon terminals of CF3 $(\sim 90 . \mathrm{kHz})$ ICC neurons. Although no responses to -90 $\mathrm{kHz}$ signals were recorded in the deposit penetration, the $\mathrm{CF} 3$ representation in ICC projects just medially to this area of MGB and may have been within the deposit zone (Wenstrup et al., 1994).

In the whole of the anterolateral division, we counted 18 labeled cells (Fig. 6, Table 1). Only 12 were in the caudal part, an observation in agreement with the absence of $48-59 \mathrm{kHz}$ responses throughout the penetration. Even fewer (six, $1 \%$ of ICC labeling) were in the rostral part that includes the FMl representation. Since other parts of the ICC were strongly labeled, it is unlikely that the lack of labeling here resulted from poor sensitivity. Thus, while FMl signals strongly influenced the responses of neurons at the center of the MGB deposit site, the results do not indicate even moderate input from the FMI representation in the ICC.

$I C C$ inputs to FM-FM regions in the MGB. In the three FMFM experiments illustrated below, deposit sites extended successively more into regions along the perimeter of the MGB known to receive $24-31 \mathrm{kHz}$ input.

In the first FM-FM experiment, a WGA-HRP deposit was placed at an FM1-FM2 recording site in the rostral pole nucleus (Fig. $7 G$ ). This deposit was restricted, with tracer spread limited to the rostral pole nucleus. We recorded single- and multipleunit FM-FM responses in both the dorsal and rostral pole nuclei, 

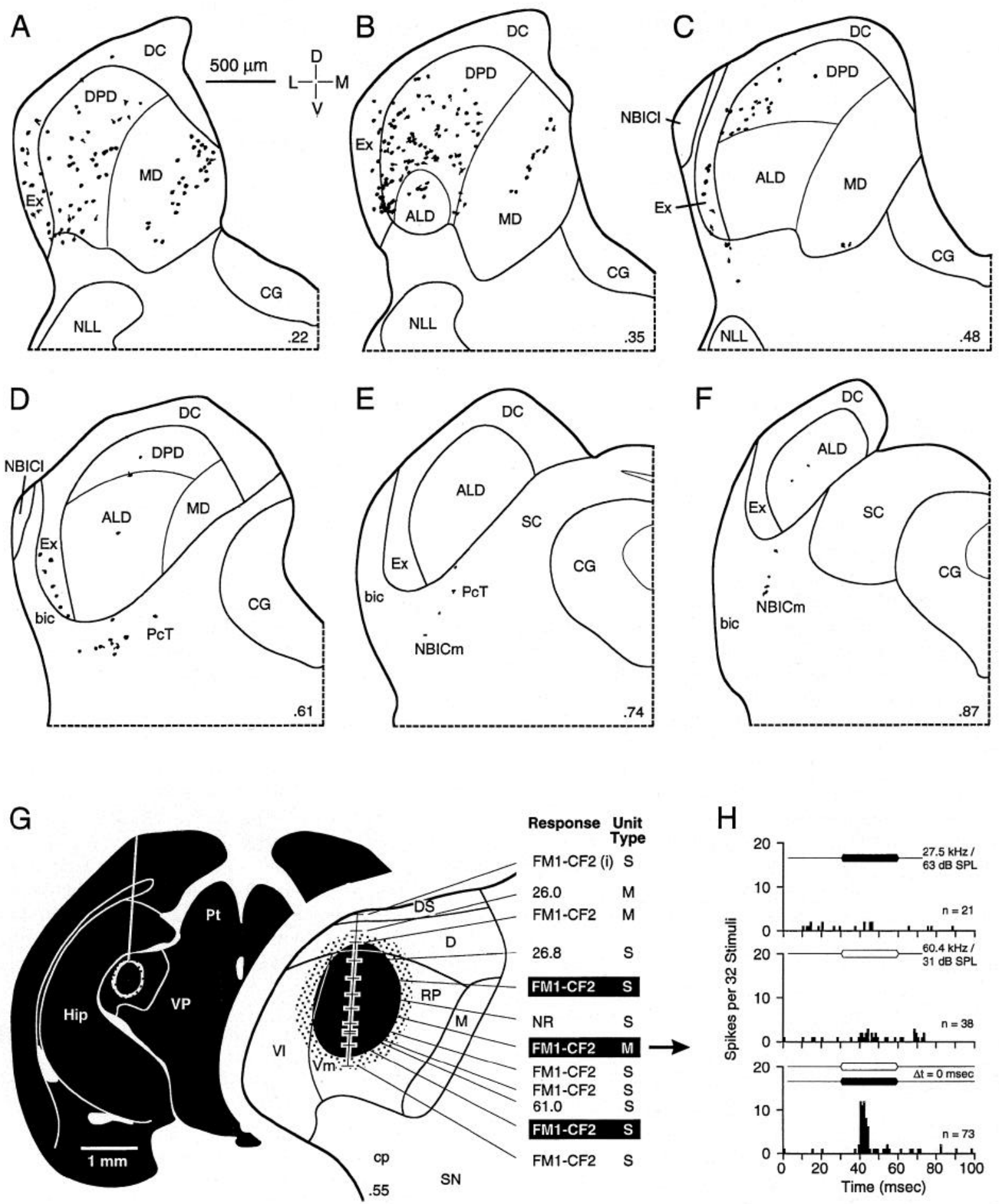

Figure 6. Results of FM1-CF2 experiment in the MGB. $A-F$, Retrograde labeling in the ICC and other midbrain regions after WGA-HRP deposit in the MGB. Dots show labeled cells in tetramethylbenzidine (TMB)-processed sections. Most labeled cells were in the dorsoposterior division $(D P D)$, the medial division $(M D)$, or the external nucleus of the inferior colliculus $(E x)$. Very few cells were labeled in the rostral part of the anterolateral division $(A L D)$, where the $24-31 \mathrm{kHz}$ representation lies. G, Physiological properties and deposit site in the MGB. Solid black region shows core of deposit site, stippled region indicates our estimate of zone of transport. White-on-black lettering shows response properties at WGAHRP deposit sites. The deposit zone was mostly limited to the medial part of the ventral division $(\mathrm{Vm})$, where many single-unit and multiunit responses were combination sensitive. The duration of iontophoresis in this experiment was equivalent to a single deposit (4.5 min), but the electrode was moved up and down by $200 \mu \mathrm{m}$ to create a more elongated deposit zone. Under Response, we indicate the type of combination-sensitive response (e.g., $F M 1-C F 2$ ), the best frequency of singly tuned neurons, or no auditory response $(N R)$. An $(i)$ indicates that the interaction between the two components was inhibitory. Under Unit Type, $(S)$ indicates single-unit, and $(M)$ indicates multiunit response. H, peristimulus time (PST) histograms showing strong facilitation in responses to combination of tones in the FM1 and CF2 frequency ranges, obtained at the middle deposit site (arrow). 
Table 1. Distribution of labeled cells in ICC subdivisions after tracer deposits in combinationsensitive regions of the MGB

\begin{tabular}{|c|c|c|c|c|c|}
\hline \multirow[b]{2}{*}{ Experiment } & \multirow{2}{*}{$\begin{array}{l}\text { Total } \\
\text { ICC } \\
\text { labeled } \\
\text { cells }\end{array}$} & \multicolumn{4}{|l|}{$n(\%)$} \\
\hline & & $\mathrm{ALDr}^{a}$ & $\mathrm{ALDc}^{a}$ & DPD & $\mathrm{MD}$ \\
\hline FM1-CF2 (Fig. 6) & 546 & $6(1.1)$ & $12(2.2)$ & $374(68.5)$ & $154(28.2)$ \\
\hline FM1-FM2 (Fig. 7) & 270 & $0(0)$ & $119(41.1)$ & $10(3.7)$ & $141(52.2)$ \\
\hline FM1-FM4 (Fig. 8) & 551 & $20(3.6)$ & $59(10.7)$ & $24(4.4)$ & $448(81.3)$ \\
\hline FM1-FM4 (Fig. 9) & 706 & $197(27.9)$ & $168(23.8)$ & $31(4.4)$ & $310(43.9)$ \\
\hline FM1-FM3 (Fig. 11) & 551 & $19(3.4)$ & $193(35.0)$ & $62(11.3)$ & $277(50.3)$ \\
\hline
\end{tabular}

"The anterolateral division was divided into the rostral part (ALDr) and the caudal part (ALDc) as described and justified in Results. They are not cytoarchitectonic subdivisions.

and their physiological properties varied. Dorsally, the FM1 effect was inhibitory, while more ventral neurons had both facilitatory and inhibitory FM1 influences and more restricted tuning to the delay between FM1 and FMn signals (Fig. $7 G$ ). Units at the deposit site responded best to an FM2 delay of 6 msec (Fig. $7 H$ ). Dorsal and ventral to the deposit site, most other neurons were both combination and delay sensitive.

Retrograde ICC labeling was highly restricted (Fig. 7A-F, Table 1). Many labeled cells $(119,41 \%)$ were in the caudal part of the anterolateral division (Fig. $7 A-C$ ), extending rostrally as far as 0.60 of the caudal-to-rostral dimension. Here, the anterolateral division contains neurons responding to the FM2 frequency range (Fig. 5). More cells $(141,52 \%)$ were in the medial division of the ICC (Fig. $7 A$ and more caudal sections). Their distribution corresponds to the tonotopic representations of the FM3 and CF3 sonar components (O'Neill et al., 1989; Wenstrup et al., 1994; Fig. 1B,C). Such labeling was expected, since these frequencies were recorded within the extent of the MGB deposit. In contrast, we recorded no $\mathrm{CF} 2$ responses, and labeled cells in the dorsoposterior division of the ICC were correspondingly very few. Thus, labeling in high-frequency ICC representations was very specific for those frequency bands recorded within the deposit zone.

No labeled cells were found in the rostral part of the anterolateral division (Fig. $7 D-F$ ), despite the strong facilitating effect of FM1 signals. Since we have recorded ICC neurons with best frequencies in the $\mathrm{FM} 1$ range $(24-29.5 \mathrm{kHz})$ only in the rostral $32 \%$ of the IC (Fig. 2), this MGB deposit apparently did not label neurons in the FM1 tonotopic representation of ICC. Furthermore, because labeled cells in other ICC regions were common (270 cells), the lack of FM1 labeling is probably not the result of poor sensitivity.

In a second FM-FM experiment, we placed a WGA-HRP deposit at a site showing FM1-FM4 responses (Fig. 8G). This deposit site was larger than in Figure 7, spanning the rostral pole and dorsal nuclei and extending to the dorsal surface of the MGB. Throughout the penetration, combination-sensitive FM1$\Gamma \mathrm{M} n$ responses were obtained. All had best delays exceeding 3 msec. Although delay tuning was not obtained at the deposit site, FM1-FM4 responses on either side of the deposit site had long best delays ( $8-12$ msec; Fig. $8 H$ ).

Most retrograde labeling of the ICC was in its medial division (448 cells, 81\%), distributed in two distinct foci (Fig. 8A-C, Table 1). One was in the ventromedial extreme, presumably corresponding to the FM4 representation. The other formed a dorsomedial to ventrolateral band placed more laterally; this is probably the FM3 representation. A third, smaller focus of label was in the caudal part of the anterolateral division (Fig. 8B,C) where FM2 frequencies are represented. Together, these foci accounted for $92 \%$ of ICC labeling, demonstrating the specificity of the labeling for ICC regions that represent the higher sonar harmonics recorded within the deposit zone. Furthermore, the greatest number of labeled ICC cells was in the medial division, which represents FM3 and FM4 frequencies nearer the center of the deposit site.

In the rostral part of the anterolateral division, only a few cells were labeled $(20,3.5 \%$ of ICC labeling); all are shown in Figures $8 D-F$. Of these, many in Figure $8 D$ are probably FM2 neurons, based on their dorsoventral and rostrocaudal locations (compare to Fig. $3 I$ ). However, those in Figure $8, E$ and $F$, may lie in the CF1 or FM1 representations. Because the deposit site extended to the dorsal surface of the MGB, a region known to receive ICC input from FM1 or CF1 representations (Wenstrup et al., 1994; Fig. 2E, this study), some labeling in these ICC representations was expected. Thus, it is probable that even fewer of the 20 labeled cells are neurons in the FM1 representation of ICC that project to the combination-sensitive region at the center of the deposit site. Here, as in the experiments above, FM1 signals strongly influenced the responses of neurons within the deposit zone, but there was not even moderate input from the FM1 representation in the ICC. Table 1 also shows similar labeling in the rostral part of the anterolateral division from an FM1-FM3 experiment, the deposit site illustrated in Figure 11.

In the third illustrated FM-FM experiment, a WGA-HRP deposit was placed at a site showing FM1-evoked inhibition of FM4 responses (Fig. 9G). This experiment was our "worst" case because the deposit zone was the largest, extending into and filling the adjacent $\mathrm{Vl}$ in the rostral part of the MGB. V1 receives strong input from ICC representations of the FM1 and CF1 sonar components (Wenstrup et al., 1994; Fig. 2, this study), and a V1 deposit should label $24-31 \mathrm{kHz}$ representations in the ICC. Several units in this penetration showed sensitivity to both FM1 and higher harmonic FM signals. At the deposit site, the predominant effect of stimuli in the FM1 frequency range was inhibition (Fig. 9G,H).

This deposit labeled more ICC cells than in the other FM-FM experiments, probably the result of the larger deposit. Most retrograde labeling occurred in the medial and anterolateral divisions (Fig. 9A-F). The medial division labeling agreed with recordings of FM3 and FM4 responses within the deposit zone. In the caudal part of the anterolateral division (Fig. 9C,D), cells could have been labeled by transport from either FM1-FM2 
A

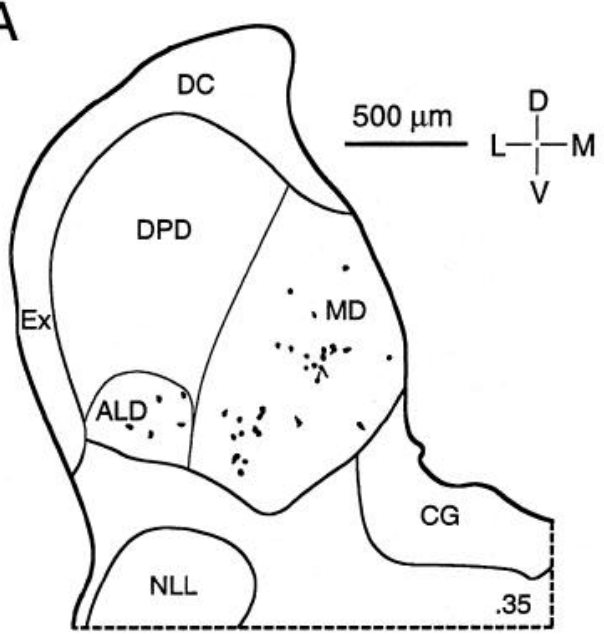

D

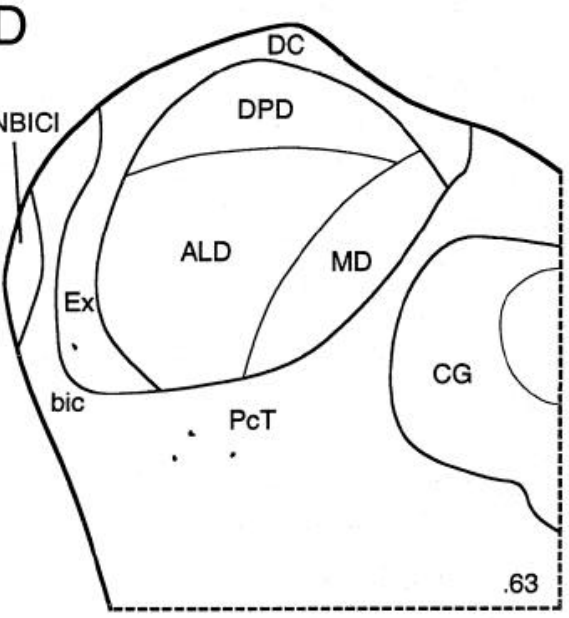

$\mathrm{B}$

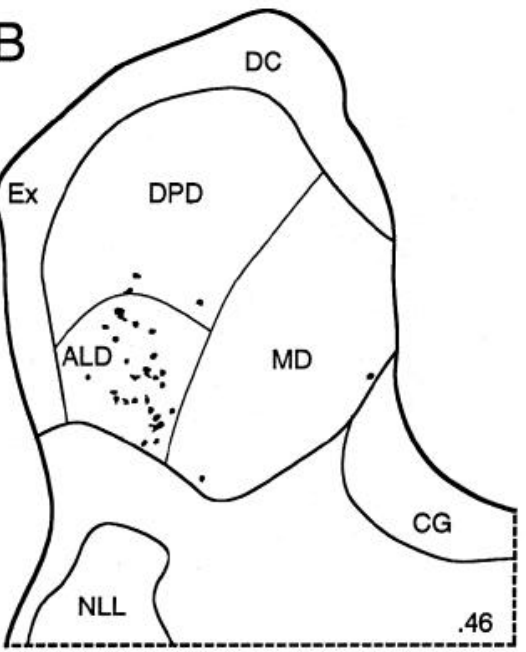

$E$

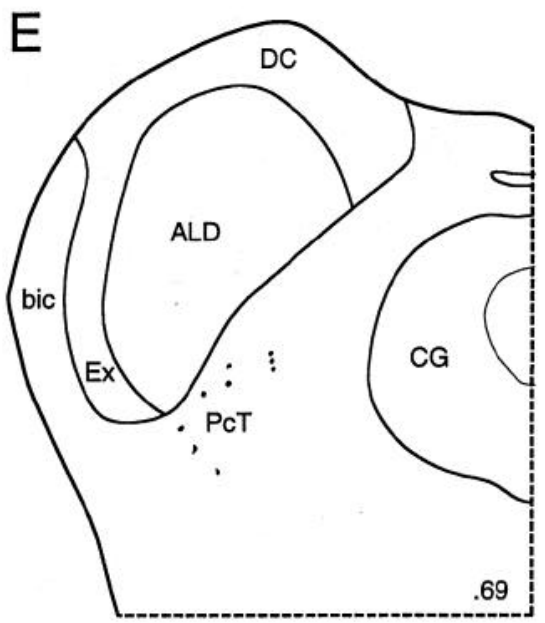

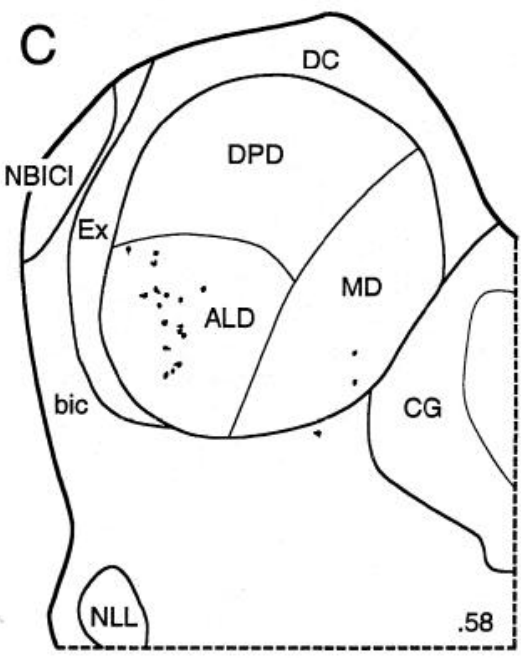

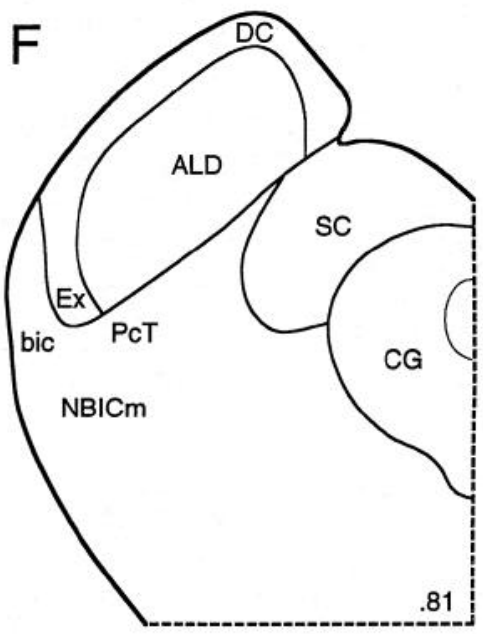

$\mathrm{H}$

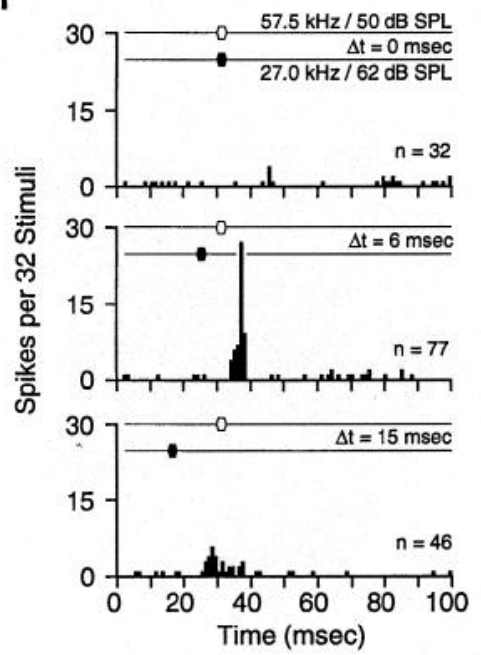

Figure 7. Results of FM1-FM2 experiment in the MGB with highly restricted deposit site. $A-F$, Retrogradely labeled cells in the ICC and other midbrain regions after WGA-HRP deposit in the MGB. Most labeled cells were in the caudal part of the anterolateral division ( $A L D)$ and in the medial division $(M D)$. No labeled cells were in the rostral part of the ALD. G, Physiological properties and deposit site in the MGB. The deposit zone was limited to the rostral pole nucleus $(R P)$. Several responses in the vicinity of the deposit site were combination sensitive. $H$, PST histograms showing delay-dependent facilitation at deposit site (arrow). Same protocol as in Figure 6. 

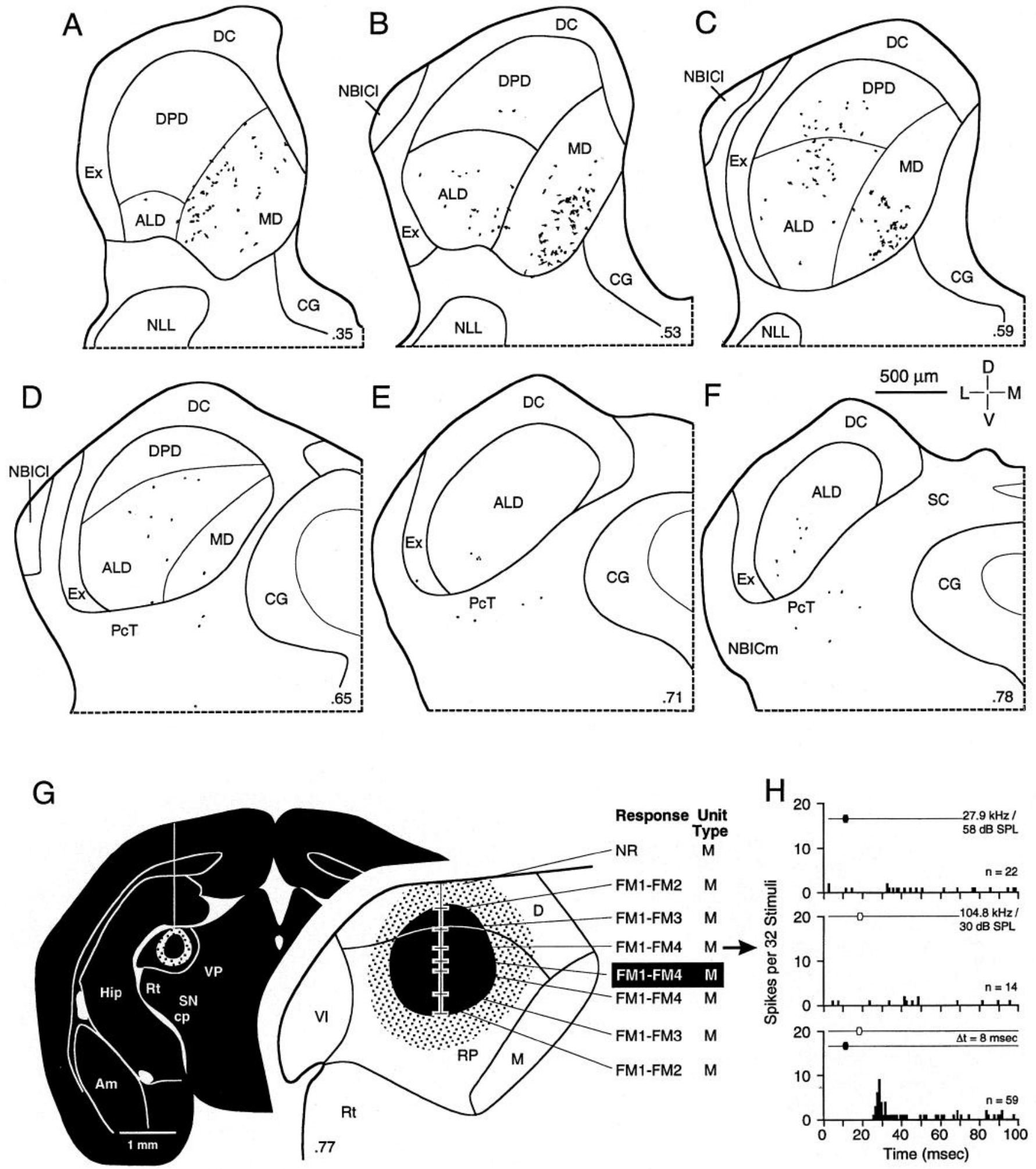

Figure 8. Results of FM1-FM4 experiment in the MGB with moderately sized deposit site. $A-F$, Retrogradely labeled cells in the ICC and other midbrain regions after WGA-HRP deposit in the MGB. Most labeled cells were in the caudal part of the anterolateral division (ALD) and in the medial division $(M D)$; a few labeled cells were in the rostral part of the ALD. G. Physiological properties and deposit site in the rostral pole (RP) and dorsal $(D)$ nuclei of the MGB. The size of this deposit was larger than that shown in Figure 7 , including the dorsal extreme of the dorsal division, a region that receives $24-31 \mathrm{kHz}$ input. This may have resulted in more labeling in the rostral ALD. All acoustically responsive cells in this penetration were FM-FM neurons. $H$, PST histograms showing facilitation in response to combination of tones in the FM1 and FM4 frequency ranges, obtained at recording site adjacent to deposit site (arrow). Same protocol as in Figure 6. 
A

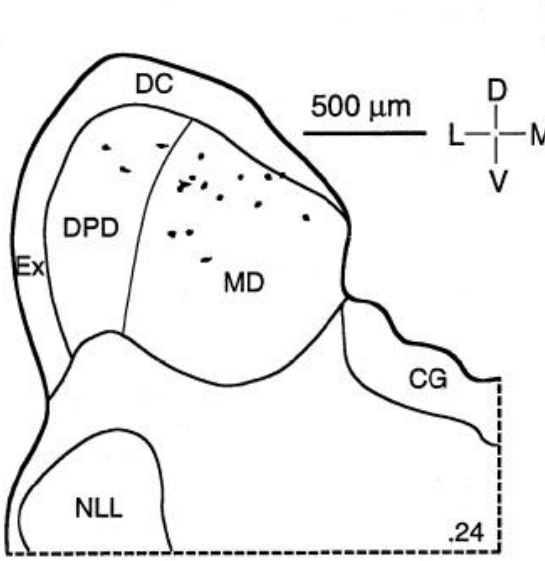

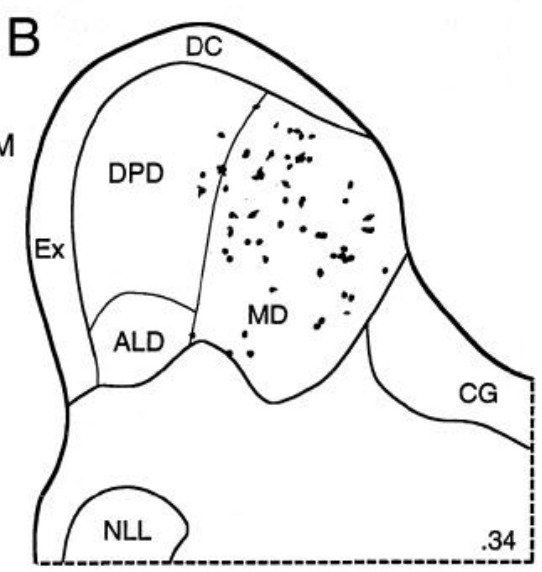

.34
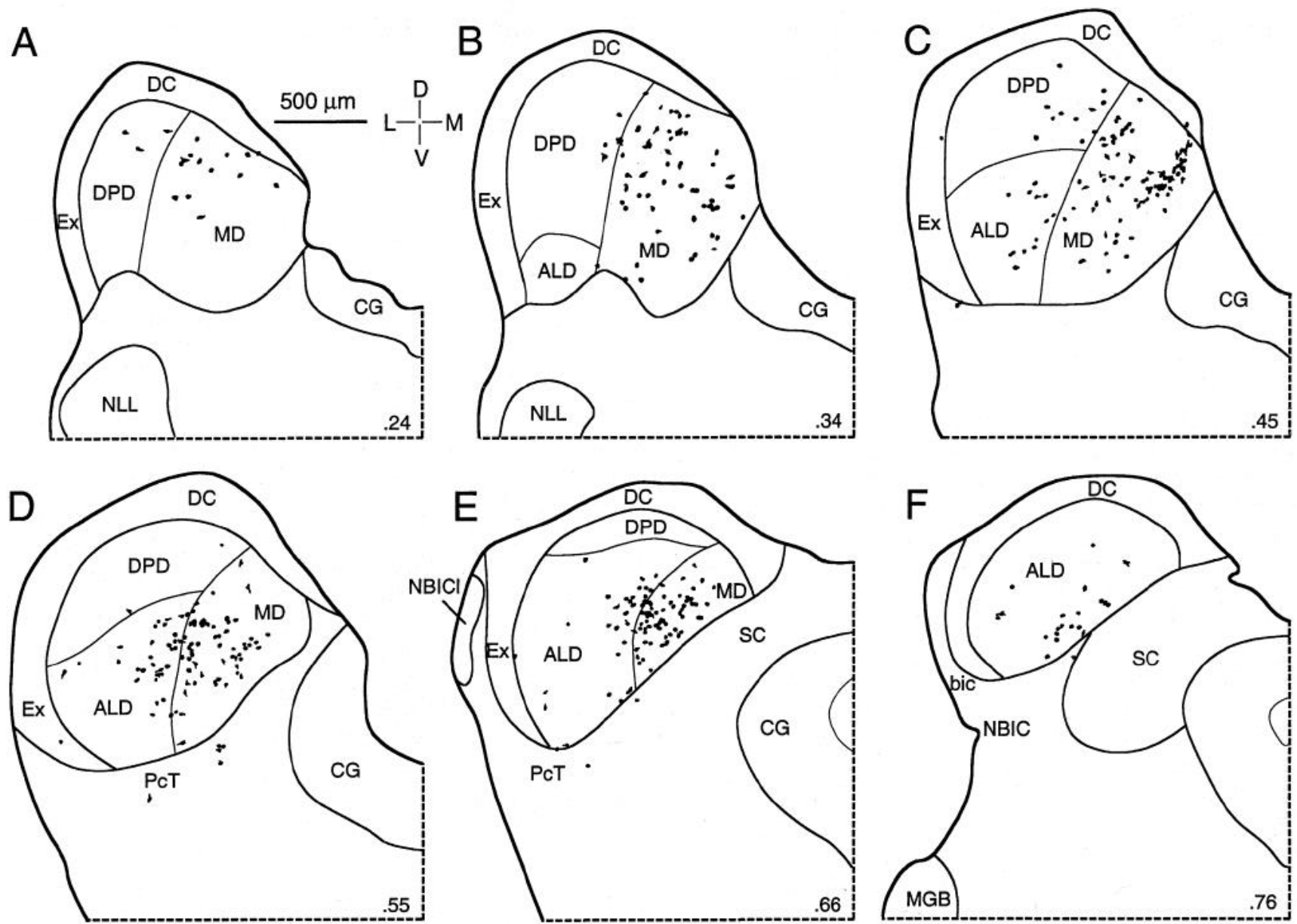

G
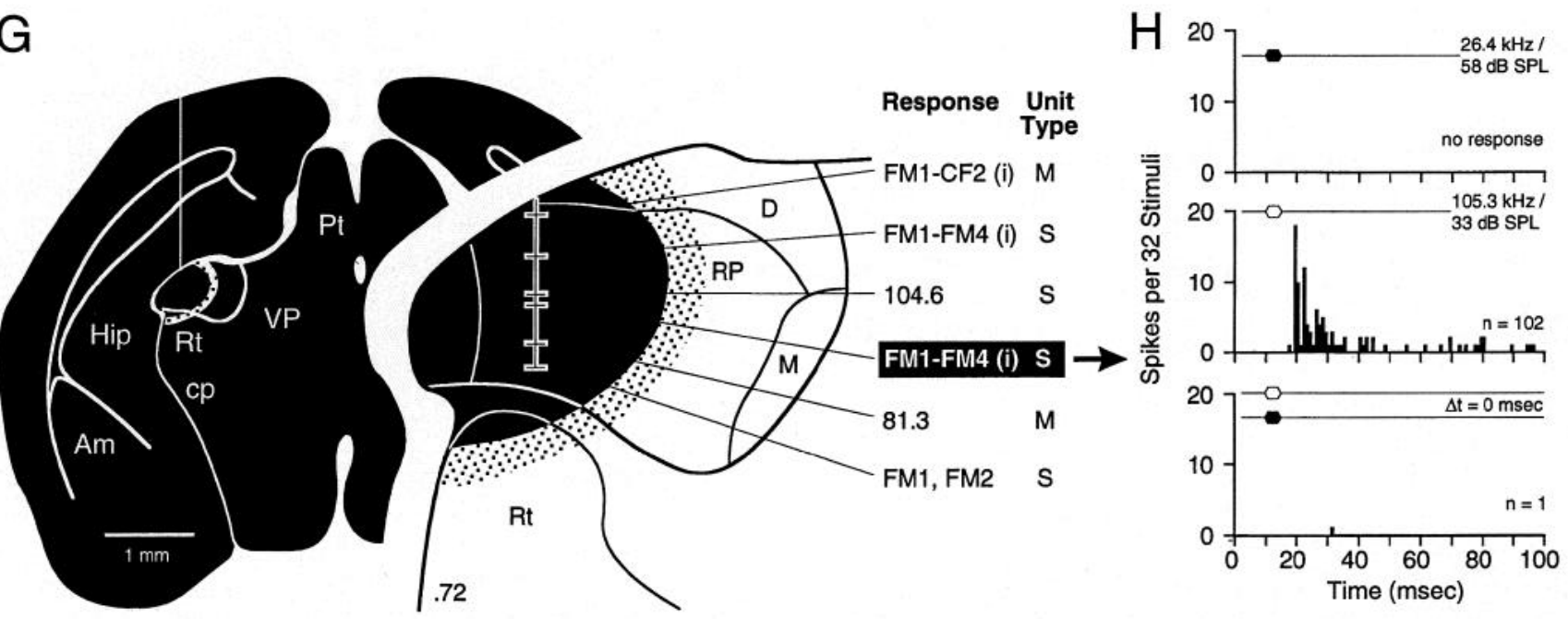

Figure 9. Results of FM1-FM4 experiment in the MGB with large deposit site that included the lateral part of the ventral division ( Vl). $A-F$, Retrogradely labeled cells in the ICC and other midbrain regions after WGA-HRP deposit in the MGB. Cells are labeled at all rostrocaudal levels of the anterolateral division $(A L D)$ and the medial division $(M D)$. The total ICC labeling was greater than in other experiments. $G$, Physiological properties and deposit site in the MGB. This deposit, larger than that in Figure 8, included the rostral pole $(R P)$ nucleus and the lateral part of the ventral division $(\mathrm{Vl})$ rostrally. Transport from $\mathrm{Vl}$ is probably responsible for the larger number of labeled cells in the rostral ALD. $H$, PST histograms showing FM1 inhibition of the excitatory response to tones in the FM4 frequency range, obtained at the deposit site (arrow). Same protocol as in Figure 6. 
Table 2. Distribution of labeled cells in all brain regions after tracer deposits in combinationsensitive regions of the MGB

\begin{tabular}{llllll} 
& $\begin{array}{l}\text { Total } \\
\text { labeled } \\
\text { Experiment }\end{array}$ & $n(\%)$ & & & \\
\cline { 3 - 6 } & cells & ICC & Cortex & \multicolumn{1}{c}{ Rt } & Pericollicular \\
\hline FM1-CF2 (Fig. 6) & 1119 & $546(48.8)$ & $253(22.6)$ & $180(16.1)$ & $140(12.5)$ \\
FM1-FM2 (Fig. 7) & 367 & $270(73.6)$ & $45(12.3)$ & $31(8.4)$ & $21(5.7)$ \\
FM1-FM4 (Fig. 8) & 1281 & $551(43.0)$ & $639(49.9)$ & $76(5.9)$ & $15(1.2)$ \\
FM1-FM3 (Fig. 11) & 1566 & $551(35.2)$ & $762(48.7)$ & $204(13.0)$ & $49(3.1)$ \\
\hline
\end{tabular}

a FM1-FM4 experiment in Figure 9 is omitted due to inadvertent tracer deposit at the cortical entry site and extension of main deposit into the thalamic reticular nucleus (Rt).

regions recorded within the penetration, or from the part of V1 included within the deposit zone.

Strong labeling in the rostral part of the anterolateral division ( $28 \%$ of labeled ICC cells) distinguished this experiment from nearly all others. This labeling, we believe, resulted from inclusion of $\mathrm{Vl}$ in the deposit zone, not from retrograde transport originating in the combination-sensitive region recorded in the penetration. Indeed, we only observed such labeling in experiments where deposit zones extended well into Vl. The deposit in this experiment was thus too extensive to provide a test of the MGB convergence hypothesis. It is, nevertheless, important because it shows that MGB tracer deposits can label many cells of the rostral part of the anterolateral division, if the deposit includes V1.

Summary of $M G B$ tracer experiments. There were three major results of these experiments. First, we consistently observed strong labeling in ICC divisions representing second, third, or fourth harmonic sonar components. One or another of these divisions always contained the largest number of retrogradely labeled cells after combination-sensitive MGB deposits (Table 1). Second, this labeling was specific. We found close agreement between the high frequencies recorded within the MGB deposit zones and the distribution of labeled cells within ICC divisions. Third, and most significant, FM1 and CF1 tonotopic representations of the ICC were unlabeled or weakly labeled after retrograde tracers were placed in and mostly limited to combination-sensitive regions of the MGB (Figs, 6-8). These experiments therefore show strong input to combination-sensitive neurons in the MGB from ICC neurons representing higher harmonic sonar components, but not from ICC neurons with best frequencies in the FM1 and CFI range. This last result is contrary to our expectations of the MGB convergence hypothesis.

\section{Part 3: other connections}

The results described above suggest that other sources of FM1 and CF1 input to combination-sensitive MGB neurons should be considered. Below are described additional findings of these experiments that address other potential sources of low-frequency input.

Other ICC outputs. The ICC projected to many nuclei other than the MGB. These may contribute in some way, currently unknown, to circuits that create combination-sensitive neurons in the MGB. Other regions labeled anterogradely by $24-31 \mathrm{kHz}$ deposits included the pontine gray (strong), the pretectum (weak), and the contralateral inferior colliculus (weak to moderate). Regions labeled after the higher frequency deposits include the pontine gray (strong), the pretectum (strong for FMn representations), the external nucleus, pericollicular tegmentum, and/or the nucleus of the brachium of the inferior colliculus (moderate-to-strong), and the contralateral inferior colliculus (weak to moderate). These results support previous studies using other tracers (Frisina et al., 1989; Wenstrup et al., 1994).

We examined the thalamic reticular nucleus for labeling after ICC deposits, because results described below showed that it projects to MGB combination-sensitive regions. Although we occasionally saw ICC terminals along the margin of the thalamic reticular nucleus, there was no evidence of significant input from any frequency representation in ICC.

Other inputs to combination-sensitive regions in the $M G B$. In addition to retrograde labeling from the high-frequency representations of the ICC, there were three other consistent sources of input to combination-sensitive MGB regions (Table 2). The strongest of these always originated in the auditory cortex (Fig. 10), mostly from cells in layer VI (Fitzpatrick and Henson, 1994). The percentagc of total labclcd cells in auditory cortex was highly variable, accounting for $12-50 \%$ of the total number of labeled cells in different experiments. The location of cortical labeling varied with the particular type of combination sensitivity at the deposit site. FM1-CF2 deposits labeled auditory cortical regions laterally, perhaps in the $60 \mathrm{kHz}$ representation of primary auditory cortex (Fig. 10A), a region showing facilitative responses to FM1 and CF2 inputs (Fitzpatrick et al., 1993). After FM-FM deposits, labeled cortical cells were place more dorsally, in the region nearer to the lateral sulcus (Fig. 10B,C). This region is associated with FM-FM response properties in the auditory cortex, outside the tonotopic region (Suga et al., 1983; Suga and Horikawa, 1986). Generally, no retrograde labeling occurred in the caudal part of auditory cortex, which represents CFl and FMI frequencies (Suga and Jen, 1976). In the two exceptions, larger deposits labeled layer VI neurons throughout much of auditory cortex.

Another input was from the thalamic reticular nucleus, which accounted for $6-16 \%$ of all labeled cells (Table 2). All MGB deposits in combination-sensitive regions labeled neurons in the caudalmost part of the this nucleus (Figs. 10, 11). Here, the thalamic reticular nucleus has a rounded shape, rather than the two- or three-cell-deep organization described in other species or seen more rostrally in this species. However, its cellular structure and immunocytochemistry (Wenstrup, in press) are in agreement with previous reports in other species (Scheibel and Scheibel, 1966; Jones, 1975; Houser et al., 1980; Rinvik et al., 1987). It is not clear whether different thalamic reticular neurons project to FM-FM versus CF-CF neurons in the MGB.

An additional input was from midbrain regions lateral and ventral to the ICC (Figs. 6-9, Table 2); these accounted for 1$13 \%$ of all retrograde labeling. Deposits in combination-sensitive 

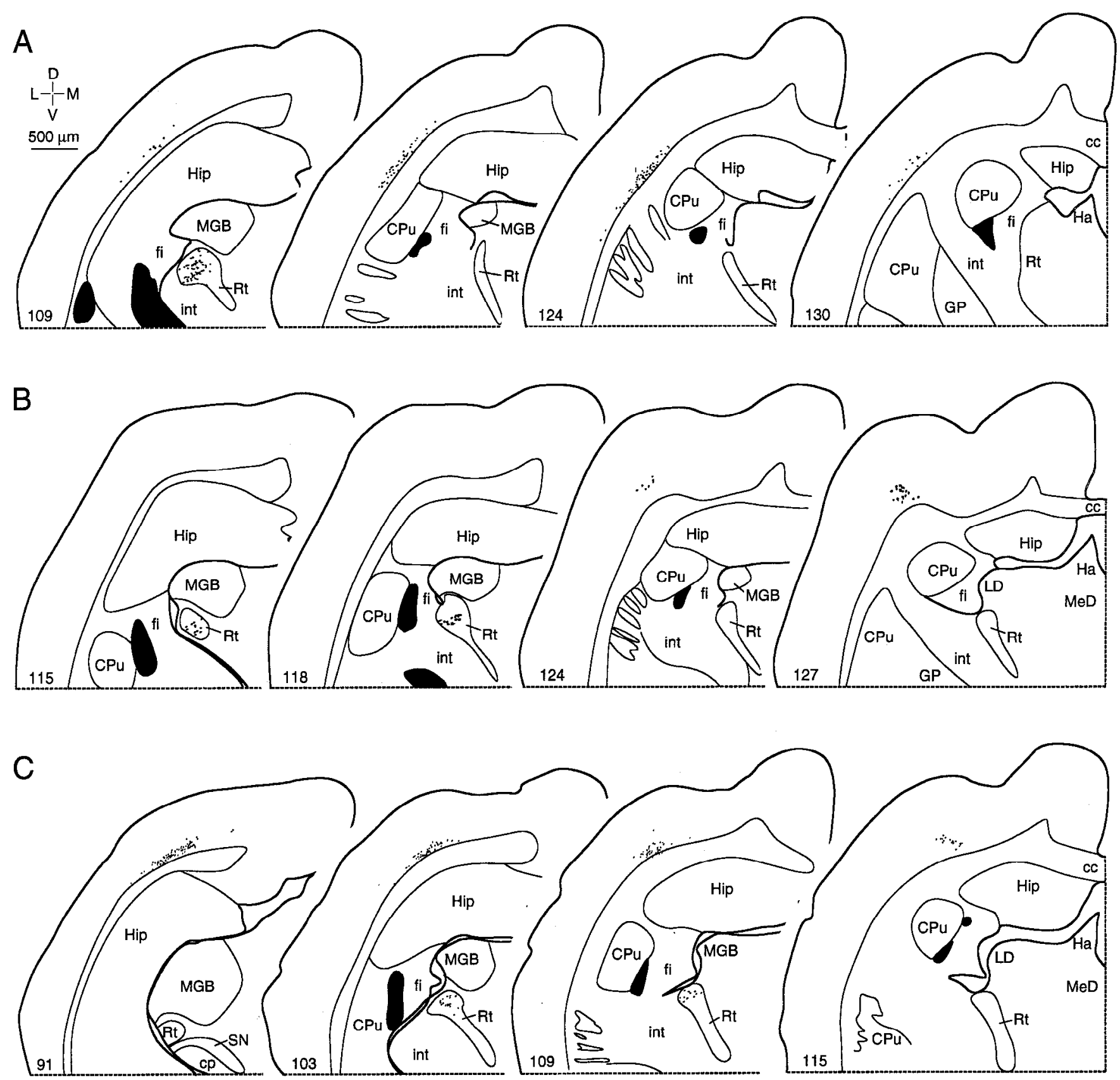

Figure 10. Retrograde labeling in the auditory cortex and thalamic reticular nucleus $(R t)$ after MGB deposits shown in Figures 6-8. A, Labeling after FM1-CF2 deposit (see Fig. $6 G$ ). Labeling was placed more ventrolaterally along the cortical surface than in FM-FM experiments. This may correspond either to the CF-CF region or to the CF2 $(60-63 \mathrm{kHz})$ representation in primary auditory cortex. $B$, Labeling after FM1-FM2 deposit (see Fig. 7G). C, Labeling after FM1-FM4 deposit (see Fig. 8G). The cortical regions labeled after FM-FM deposits were dorsal to labeling in the FM1-CF2 or CF1-CF2 regions. Numbers at lower left indicate section number, from caudal (lower numbers) to rostral.

CF2 regions of the MGB always labeled the external nucleus of the ICC and the pericollicular tegmentum, ventral to the ICC (Fig. $6 A-F$ ). Other cells were in the nucleus of the brachium of the inferior colliculus, medial part (NBICm, Fig. 6E,F). Deposits in other combination-sensitive regions, where neurons responded to FM1-FMn or CF1-CF3 signals, labeled the pericollicular tegmentum (Figs. $7 D, E ; 8 D-F ; 9 D, E$ ), but only rarely included the external nucleus lateral to the ICC.

Other auditory regions were closely examined for retrogradely labeled cells. In the MGB, there was no significant retrograde labeling outside the deposit site, including those regions that received FM1 and CF1 input from the ICC (e.g., Fig. 11). For instance, $\mathrm{Vl}$ contained no retrogradely labeled neurons if the deposit site was sufficiently distant from it. Likewise, other known targets of $24-31 \mathrm{kHz}$ ICC input, including the suprageniculate nucleus and the ventromedial extreme of the rostral pole nucleus (Wenstrup et al., 1994; Fig. 2, this study), were not retrogradely labeled. These results suggest that combination-sensitive MGB neurons do not receive $24-31 \mathrm{kHz}$ input from the ICC by way of a synaptic relay through other MGB subdivisions.

We also saw no retrograde labeling in the auditory brainstem, 


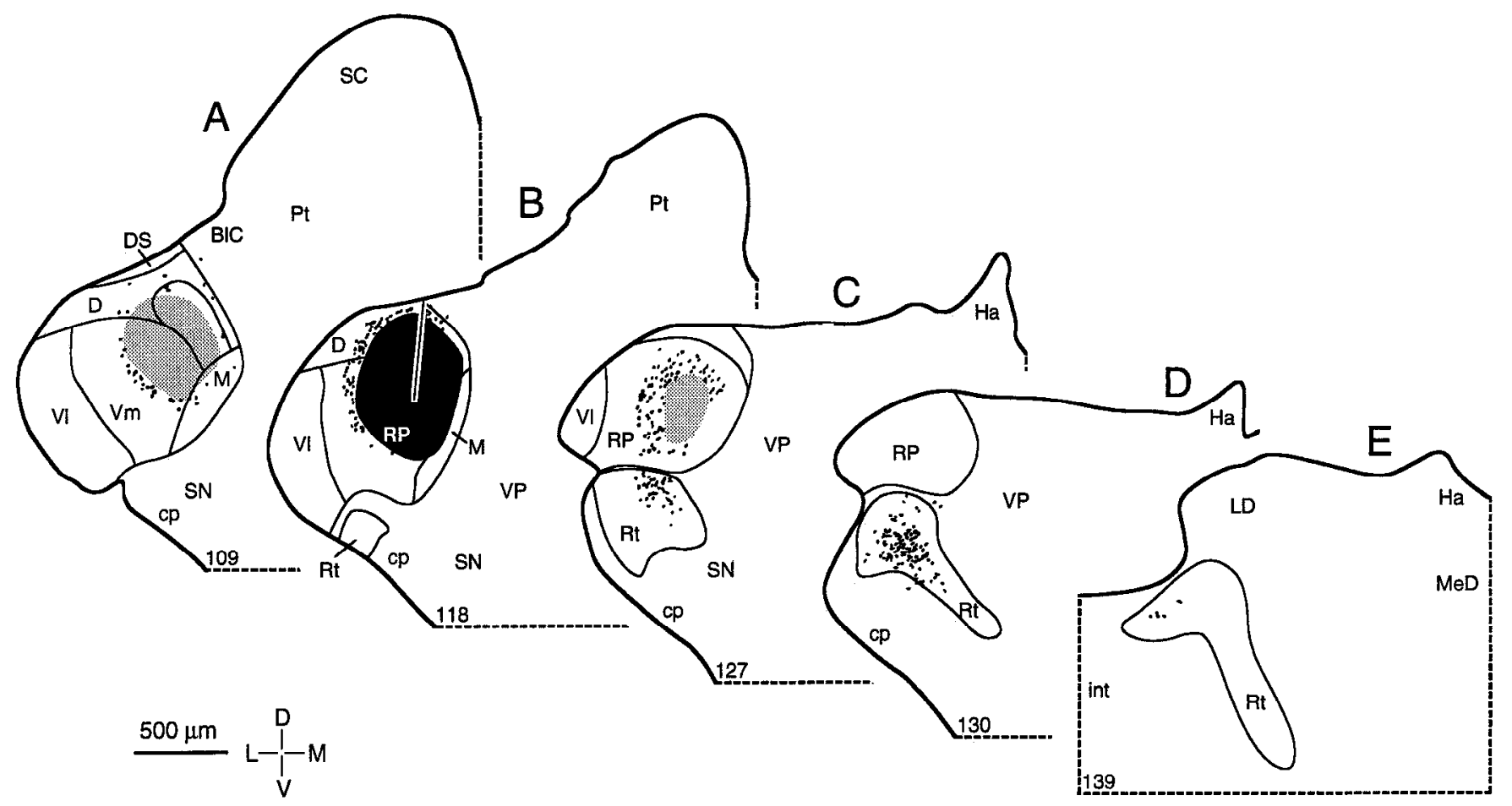

Figure 11. A-E, Thalamic retrograde labeling after FM1-FM3 deposit in MGB. Deposit site (in black) is in B. Stippling in $A$ and $C$ shows extracellular reaction product at the margins of the deposit zone that obscured cellular labeling. In the MGB, labeled cells occurred only near the deposit site. Labeled cells were abundant in the thalamic reticular nucleus (Rt), particularly in its most caudal and dorsal parts. In the ICC (not shown, see Table 1), nearly all labeled cells were in the medial division and caudal part of the anterolateral division, with very few in the rostral part of the anterolateral division. Numbers at lower left indicate section number, from caudal (lower numbers) to rostral.

including the nuclei of the lateral lemniscus and the nucleus of the central acoustic tract. These regions have been implicated in projections bypassing the inferior colliculus, but they do not project directly to the combination-sensitive regions of the MGB described here. Retrograde labeling in the nucleus of the central acoustic tract has been observed when deposits are placed in the suprageniculate nucleus of the mustached bat (Casseday et al., 1989).

\section{Discussion}

This study examined circuitry that contributes to the combination-sensitive response of MGB neurons believed to encode biosonar target information. In both anterograde and retrograde tracing experiments, we found no consistent evidence of a projection from ICC neurons within the tonotopic $24-31 \mathrm{kHz}$ representation to many regions of the medial geniculate body containing combination-sensitive neurons. These results question the validity of the MGB convergence hypothesis, and suggest that the low-frequency sensitivity of combination-sensitive MGB neurons may depend on other sources. Additional findings concerned other potential sources, which include the high-frequency representations of the inferior colliculus, the external nucleus of the IC and pericollicular tegmentum, the thalamic reticular nucleus, and auditory cortex. These findings may require a change in our understanding of the locations or the mechanisms by which combination-sensitive neurons are formed.

\section{A negative conclusion}

The general assumption that combination-sensitive neurons are formed in the MGB is based on physiological evidence showing that these neurons exist in the MGB (Olsen and Suga, 1991a,b) but apparently not in the ICC (O'Neill, 1985). Because the influence of $\mathrm{CF} 1$ or FM1 signals on responses in the rostral half of the MGB is both pervasive and strong, it seems reasonable to expect that neurons in ICC representations of the biosonar fundamental $(24-31 \mathrm{kHz})$ should project in moderate strength throughout the rostral MGB. A previous study was unable to find such a projection (Wenstrup et al., 1994).

Recognizing the difficulty in showing the absence of a neural connection, we used both anterograde and retrograde tracing with physiological recording to correlate the response properties of ICC and MGB neurons with their connections. While our conclusions rely more heavily on the retrograde tracer experiments, there was a high degree of consistency in the results from the two techniques and between these results and those of the previous study (Wenstrup et al., 1994). For example, each showed that ICC representations of the fundamental project mainly to MGB regions along its perimeter, but not to the central part that includes $\mathrm{Vm}$, the rostral pole nucleus, and part of the dorsal nucleus (Figs. 2, 6-9; Wenstrup et al., 1994).

We occasionally found FM1 or CF1 labeling in the ICC (after combination-sensitive MGB deposits) and in the combinationsensitive MGB (after 24-31 kHz ICC deposits), but this labeling was generally weak and inconsistent. We also presented experiments that showed apparently strong $24-31 \mathrm{kHz}$ labeling (Figs. 3,9 ), but such labeling occurred only when deposits included unintended high-frequency regions of the ICC (in anterograde experiments) or nuclei along the margin of the MGB that clearly receive $24-31 \mathrm{kHz}$ input (in retrograde experiments). These latter experiments strengthen our conclusions because their inadvertent labeling patterns are predicted by the findings of our other experiments. 
The present results do not rule out the possibility that dendrites of combination-sensitive neurons might extend very far, $250 \mu \mathrm{m}$ or more from the cell bodies, to sample $24-31 \mathrm{kHz}$ ICC input from zones along the margin of the MGB. However, the dendritic arborizations seen in Golgi-impregnated MGB neurons do not support this hypothesis (Winer and Wenstrup, 1994b), particularly in $\mathrm{Vm}$ where the Golgi data are strongest and where neurons are facilitated by FM1-CF2 combinations. Dendrites in Vm rarely extend more than 120-150 $\mu \mathrm{m}$ from the soma.

Thus, after two anatomical studies examining ICC inputs to the MGB, there is no evidence of moderate or strong 24-31 $\mathrm{kHz}$ input to many combination-sensitive neurons in the MGB, particularly in Vm and the rostral pole nucleus. While some MGB combination-sensitive neurons, particularly in nuclei along the margin of the MGB, may be formed by ICC convergence and others may be weakly influenced by $24-31 \mathrm{kHz}$ input from the ICC, our view is that ICC input from these representations cannot explain the widespread $24-31 \mathrm{kHz}$ sensitivity of neurons in the rostral half of the MGB. Other sources of $24-31 \mathrm{kHz}$ influence must be important and we consider them below.

\section{Sources of $24-31 \mathrm{kHz}$ input}

If ICC representations of $24-31 \mathrm{kHz}$ do not project to combination-sensitive MGB neurons, than some other source provides low-frequency input or the MGB receives input from a source that is combination sensitive. Our results in conjunction with other recent evidence suggest that each may occur. Below, we consider what evidence supports these possibilities.

Input from high-frequency ICC representations. The largest ascending input to MGB combination-sensitive regions originated in high-frequency representations of the ICC. The topographic projection pattern of these ICC representations (Frisina et al., 1989; Wenstrup et al., 1994; this study) is consistent with the topographic distribution of higher harmonic sensitivities among MGB combination-sensitive neurons (Olsen and Suga, 1991a; this study). Thus, ICC representations of FM2, FM3, and FM4 sonar components send strong projections to the rostral third of the MGB, where FM-FM neurons predominate, while most FM-CF and CF-CF neurons were recorded in the middle one-third of the MGB, which receives the strongest $\mathrm{CF} 2$ and CF3 input.

A recent study indicates that many neurons in these highfrequency representations of the ICC are combination sensitive (Mittmann and Wenstrup, 1994). In fact, the majority of highfrequency ICC neurons tested displayed some influence of signals in the $24-31 \mathrm{kHz}$ range. Some were only inhibited by these low-frequency signals, while others showed typical FMFM, FM-CF, or CF-CF facilitative responses found in the MGB or auditory cortex. It is too early to know whether such neurons are the exclusive source of combination sensitivity among MGB neurons, but it seems likely that they contribute significantly. Those results provide an explanation for the surprising lack of $24-31 \mathrm{kHz}$ input that we demonstrated here. However, it is also possible that some ICC neurons that are not combination sensitive also project to combination-sensitive MGB regions.

Other midbrain sources. Additional ascending input originates in the midbrain, mostly from the external nucleus of the IC and the pericollicular tegmentum. Although the number of neurons is small, all tracer deposits in the combination-sensitive
MGB labeled cell bodies in one or both of these regions. The external nucleus and pericollicular areas are probably sources of high-frequency input to the MGB. They receive topographically ordered projections from ICC frequency bands representing higher harmonic components of the sonar signal (Wenstrup et al., 1994; present results), and they project to corresponding combination-sensitive regions of the MGB. For example, the external nucleus of IC receives its strongest input from the 60 $\mathrm{kHz}$ (CF2) representation in ICC (Wenstrup et al., 1994) and contains sharply tuned responses to $60 \mathrm{kHz}$ signals (Wenstrup et al., 1986). In the present retrograde study, nearly all labeling of the external nucleus occurred after deposits in combinationsensitive CF2 regions of the MGB. The external nucleus and pericollicular areas may also contain combination-sensitive neurons, since they receive input from ICC regions having these properties (Mittmann and Wenstrup, 1994). However, their physiological properties, including combination sensitivity, are poorly understood.

Auditory cortex. Descending projections of auditory cortical neurons (layers V and VI) comprise a major source of input to combination-sensitive MGB regions. These neurons are probably combination sensitive, since the cortical regions labeled retrogradely in our study appear to correspond to the combinationsensitive regions described by Suga and his colleagues, including the FM-FM, CF-CF, and DSCF $(60 \mathrm{kHz}$ representation in primary auditory cortex) areas (Suga et al., 1983; Suga and Horikawa, 1986; Fitzpatrick et al., 1993). We found no consistent evidence of low-frequency input from the $24-31 \mathrm{kHz}$ representation in the caudal part of primary auditory cortex.

What is the role of this descending input? A preliminary study showed that electrical stimulation of an FM-FM cortical area exerted both excitatory and inhibitory effects on the response of combination-sensitive MGB neurons (Teng and Suga, 1994). The direct corticothalamic input is likely overwhelmingly excitatory, since corticothalamic neurons release excitatory neurotransmitters and there are very few GABAergic interneurons in the mustached bat MGB to mediate inhibition (Winer et al., 1992; Vater et al., 1992). Descending inhibitory influences are probably mediated through the thalamic reticular nucleus (see below). It is unclear whether corticothalamic projections play any role in the filter properties of MGB neurons, since normalized delay tuning, best amplitude, and response duration appear unaffected by cortical stimulation (Teng and Suga, 1994).

Thalamic reticular nucleus. The posterior, auditory sector of the thalamic reticular nucleus in the mustached bat (Wenstrup, in press) is a consistent source of input to the combinationsensitive MGB. It may also be the only source of $24-31 \mathrm{kHz}$, non-combination-sensitive input to combination-sensitive MGB regions. Preliminary physiological studies have recorded neurons exclusively responsive to frequencies in the fundamental (Olsen, personal communication; Wenstrup and Grose, 1993), although a detailed survey remains to be made. Since the thalamic reticular nucleus contains mostly GABAergic neurons in the mustached bat (Winer et al., 1992) as in other species (Houser et al., 1980; Rinvik et al., 1987), it may supply CF1 or FM1 inhibition to combination-sensitive MGB neurons. Such inhibition may be the basis for the early inhibitory influence of FM1 input that is a key feature of FM-FM neurons having long best delays (Olsen and Suga, 1991b). However, the latencies of thalamic reticular neurons have not been reported, and it is not known whether they respond at sufficiently short latencies to play a role in the formation of combination-sensitive MGB re- 
sponses. In other species, this nucleus receives input from both the MGB and auditory cortex (Jones, 1975; Conley et al., 1991). Further studies are needed of auditory responses of thalamic reticular neurons, the sources of their auditory input, and their effect on MGB combination-sensitive neurons, because this nucleus could play a role in the creation of combination-sensitive MGB neurons that require inhibitory $24-31 \mathrm{kHz}$ input.

We conclude that many combination-sensitive MGB neurons receive these response properties from ascending neurons of the ICC and nearby midbrain regions, which are themselves combination sensitive. Other MGB neurons, probably fewer, may form combination-sensitive responses from ascending high-frequency input in combination with low-frequency input from the thalamic reticular nucleus or, in some parts of the MGB, from 24-31 kHz ICC input. Thalamic reticular and/or descending, combination-sensitive cortical input may modulate the responsiveness or the selectivity of MGB neurons.

\section{Origin of delay sensitivity in FM-FM neurons}

FM-FM neurons respond selectively to the delay between an FMl signal in the outgoing pulse and a weaker FMn signal in an echo, and populations of neurons with different delay selectivities provide a neural representation of target distance (O'Neill and Suga, 1982; Suga et al., 1983). At sites of convergent frequency input, whatever the location, mechanisms of delay tuning depend on the coincidence of excitatory postsynaptic potentials from both the pulse FM1 and echo FMn (Suga et al., 1990; Olsen and Suga, 1991b). Such coincidence requires that the excitatory effect of the pulse FM1 be delayed within the nervous system, and several mechanisms have been proposed to explain aspects of the delayed FM1 excitation. Kuwabara and Suga (1993) reported that the brachial axons of FM1 ICC neurons have larger and more variable latencies than do neurons responding to other sonar components. Park and Pollak (1993), recording from $60 \mathrm{kHz}$ (CF2) neurons, found a broad distribution of latencies in the ICC. They suggested that the distribution of latencies is broad among both FM1 and FM $n$ neurons in the ICC, and that delay sensitivity results from the match of appropriately timed FM1 and FMn inputs at the level of the MGB. A third mechanism, particularly for neurons having long best delays, is that delayed FM1 excitation is a rebound from a shortlatency, FM1-induced inhibition (Suga et al., 1990; Olsen and Suga, 1991b). For each of these mechanisms, the assumption has been that MGB neurons are the coincidence detectors. However, this study and the finding of combination-sensitive neurons in the inferior colliculus (Mittmann and Wenstrup, 1994) suggest that integration may occur lower than the MGB, and that mechanisms that create delay lines may operate on brainstem inputs to the ICC and on the ICC itself.

\section{Appendix}

\section{List of abbreviations}

ALD anterolateral division of the central nucleus of the in-

Am ferior collicu

bic brachium of the inferior colliculus

cc corpus callosum

$\mathrm{CF} \quad$ constant frequency

$\mathrm{CF} n \quad n$th harmonic of constant frequency biosonar compo-

CG central gray

$\mathrm{cp} \quad$ cerebral peduncle

$\mathrm{CPu} \quad$ caudate-putamen

$\mathrm{CTb} \quad$ cholera toxin, B-subunit

$\begin{array}{ll}\text { D } & \text { dorsal nucleus of the MGB } \\ \text { DAB } & \text { diaminobenzidine } \\ \text { DC } & \text { dorsal cortex of the inferior colliculus } \\ \text { DPD } & \text { dorsoposterior division of the central nucleus of the } \\ \text { inferior colliculus }\end{array}$

\section{References}

Adams JC (1981) Heavy metal intensification of DAB-based HRP reaction product. J Histochem Cytochem 29:775.

Casseday JH, Kobler JB, Ishey SF, Covey E (1989) Central acoustic tract in an echolocating bat: an extralemniscal auditory pathway to the thalamus. J Comp Neurol 287:247-259.

Conley M, Kupersmith AC, Diamond IT (1991) The organization of projections from subdivisions of the auditory cortex and thalamus to the auditory sector of the thalamic reticular nucleus in Galago. Eur J Neurosci 3:1089-1103.

Fitzpatrick DC, Henson OW Jr (1994) Cell types in the mustached bat auditory cortex. Brain Behav Evol 43:79-91.

Fitzpatrick DC, Kanwal JS, Butman JA, Suga N (1993) Combinationsensitive neurons in the primary auditory cortex of the mustached bat. J Neurosci 13:931-940.

Frisina RD, O'Neill WE, Zettel ML (1989) Functional organization of mustached bat inferior colliculus: II. Connections of the $\mathrm{FM}_{2}$ region. J Comp Neurol 284:85-107.

Fuzessery ZM, Feng AS (1983) Mating call selectivity in the thalamus and midbrain of the leopard frog (Rana p. pipiens): single and multiunit analyses. J Comp Physiol 150:333-344.

Henson OW, Pollak GD, Kobler JB, Henson MM, Goldman LJ (1982) Cochlear microphonic potentials elicited by biosonar signals in flying bats, Pteronotus p. parnellii. Hearing Res 7:127-147.

Houser CR, Vaughn JE, Barber RP, Roberts E (1980) GABA neurons are the major cell type of the nucleus reticularis thalami. Brain Res 200:345-354.

Izzo PN (1991) A note on the use of biocytin in anterograde tracing 
studies in the central nervous system: application at both light and electron microscopic level. J Neurosci Methods 36:155-166.

Jones EG (1975) Some aspects of the organization of the thalamic reticular complex. J Comp Neurol 162:285-308.

King MA, Louis PM, Hunter BE, Walker DW (1989) Biocytin: a versatile anterograde neuroanatomical tract-tracing alternative. Brain Res 497:361-367.

Kuwabara N, Suga N (1993) Delay lines and amplitude selectivity are created in subthalamic auditory nuclei: the brachium of the inferior colliculus. J Neurophysiol 69:1713-1724.

LeDoux JE, Ruggiero DA, Reis DJ (1985) Projections to the subcortical forebrain from anatomically defined regions of the medial geniculate body in the rat. J Comp Neurol 242:182-213.

Luppi P-H, Sakai K, Fort P, Salvert D, Jouvet M (1988) The nuclei of origin of monoaminergic, peptidergic, and cholinergic afferents to the cat nucleus reticularis magnocellularis: a double-labeling study with cholera toxin as a retrograde tracer. J Comp Neurol 277:1-20.

Maekawa M, Wong D, Paschal WG (1992) Spectral selectivity of FMFM neurons in the auditory cortex of the echolocating bat, Myotis lucifugus. J Comp Physiol [A] 171:513-522.

Margoliash D, Fortune ES (1992) Temporal and harmonic combination-sensitive neurons in the zebra finch's HVc. J Neurosci 12:43094326.

Mesulam M-M (1982) Principles of horseradish peroxidase neurohistochemistry and their applications for tracing neural pathways-axonal transport, enzyme histochemistry and light microscopic analysis. In: Tracing neural connections with horseradish peroxidase (Mesulam M-M, ed), pp 1-151. New York: Wiley.

Mittmann DII, Wenstrup JJ (1994) Combination-sensitive neurons in the inferior colliculus of the mustached bat. Abstr Assoc Res Otolaryngol 17:93.

Mudry KM, Constantine-Paton M, Capranica RR (1977) Auditory sensitivity of the diencephalon of the leopard frog, Rana p. pipiens. J Comp Physiol 114:1-13.

Novick A (1963) Orientation in neotropical bats. II. Phyllostomatidae and Desmondontidae. J Mammal 44:44-56.

Olsen JF (1994) Medial geniculate neurons in the squirrel monkey sensitive to inter-component delays that categorize species-typical calls. Abstr Assoc Res Otolaryngol 17:21.

Olsen JF, Suga N (1991a) Combination-sensitive neurons in the medial geniculate body of the mustached bat: encoding of relative velocity information. J Neurophysiol 65:1254-1274.

Olsen JF, Suga N (1991b) Combination-sensitive neurons in the medial geniculate body of the mustached bat: encoding of target range information. J Neurophysiol 65:1275-1296.

O'Neill WE (1985) Responses to pure tones and linear FM components of the CF-FM biosonar signal by single units in the inferior colliculus of the mustached bat. J Comp Physiol [A] 157:797-815.

O'Neill WE, Suga N (1982) Encoding of target range and its representation in the auditory cortex of the mustached bat. J Neurosci $2: 17-31$.

O'Neill WE, Frisina RD, Gooler DM (1989) Functional organization of mustached bat inferior colliculus: I. Representation of FM frequency bands important for target ranging revealed by ${ }^{14} \mathrm{C}-2$-deoxyglucose autoradiography and single unit mapping. J Comp Neurol 284:60-84.

Park TJ, Pollak GD (1993) GABA shapes a topographic organization of response latency in the mustache bat's inferior colliculus. J Neurosci 13:5172-5187.

Pollak GD, Bodenhamer RD (1981) Specialized characteristics of single units in inferior colliculus of mustache bat: frequency representation, tuning, and discharge patterns. J Neurophysiol 46:605-620.

Rinvik E, Ottersen OP, Storm-Mathisen J (1987) Gamma-aminobutyrate-like immunoreactivity in the thalamus of the cat. Neuroscience 21:781-805.

Scheibel ME, Scheibel AB (1966) The organization of the nucleus reticularis thalami: a Golgi study. Brain Res 1:43-62.

Schnitzler H-U (1970) Echoortung bei der Fledermaus Chilonycteris rubiginosa. Z Vergl Physiol 68:25-38.

Schuller G, O'Neill WE, Radtke-Schuller S (1991) Facilitation and delay sensitivity of auditory cortex neurons in CF-FM bats, Rhinolophus rouxi and Pteronotus p. parnellii. Eur J Neurosci 3:11651181.

Suga N (1988) Auditory neuroethology and speech processing: complex-sound processing by combination-sensitive neurons. In: Auditory function: neurobiological bases of hearing (Edelman GM, Gall WE, Cowan WM, eds), pp 679-720. New York: Wiley.

Suga N (1990) Cortical computational maps for auditory imaging. Neural Networks 3:3-21.

Suga N, Horikawa J (1986) Multiple time axes for representation of echo delays in the auditory cortex of the mustached bat. J Neurophysiol 55:776-805.

Suga N, Jen PH-S (1976) Disproportionate tonotopic representation for processing CF-FM sonar signals in the mustache bat auditory cortex. Science 194:542-544.

Suga N, O’Neill WE, Kujirai K, Manabe T (1983) Specificity of combination-sensitive neurons for processing of complex biosonar signals in auditory cortex of the mustached bat. J Neurophysiol 49:15731626.

Suga N, Olsen JF, Butman JA (1990) Specialized subsystems for processing biologically important complex sounds: cross-correlation analysis for ranging in the bat's brain. Cold Spring Harbor Symp Quant Biol 55:585-597.

Takahashi T, Konishi M (1986) Selectivity for interaural time difference in the owl's midbrain. J Neurosci 6:3413-3422.

Taniguchi I, Niwa H, Wong D, Suga N (1986) Response properties of FM-FM combination-sensitive neurons in the auditory cortex of the mustached bat. J Comp Physiol [A] 159:331-337.

Teng H, Suga N (1994) Cortico-thalamic control: effect of electrical stimulation of the cortical FM-FM area on responses of thalamic FMFM neurons in the mustached bat. Abstr Assoc Res Otolaryngol 17: 86.

Vater M, Kössl M, Horn AKE (1992) GAD- and GABA-immunoreactivity in the ascending auditory pathway of horseshoe and mustached bats. J Comp Neurol 325:183-206.

Wenstrup JJ (1992) Inferior colliculus projections to the medial geniculate body: a study of the anatomical basis of combination-sensitive neurons in the mustached bat. Soc Neurosci Abstr 18:1039.

Wenstrup JJ (in press) The auditory thalamus in bats. In: Hearing by bats. Springer handbook of auditory research (Popper AN and Fay RR, eds). New York: Springer.

Wenstrup JJ, Grose CD (1993) Inputs to combination-sensitive neurons in the medial geniculate body of the mustached bat. Soc Neurosci Abstr 19:1426.

Wenstrup JJ, Ross LS, Pollak GD (1986) Binaural response organization within a frequency-band representation of the inferior colliculus: implications for sound localization. J Neurosci 6:962-973.

Wenstrup JJ, Fuzessery ZM, Pollak GD (1988) Binaural neurons in the mustache bat's inferior colliculus: I. Responses of $60-\mathrm{kHz}$ EI units to dichotic sound stimulation. J Neurophysiol 60:1369-1383.

Wenstrup JJ, Larue DT, Winer JA (1994) Projections of physiologically defined subdivisions of the inferior colliculus in the mustached bat: targets in the medial geniculate body and extrathalamic nuclei. $\mathrm{J}$ Comp Neurol 346:207-236.

Winer JA, Wenstrup JJ (1994a) Cytoarchitecture of the medial geniculate body in the mustached bat (Pteronotus parnellii). J Comp Neurol 346:161-182.

Winer JA, Wenstrup JJ (1994b) The neurons of the medial geniculate body in the mustached bat (Pteronotus parnellii). J Comp Neurol 346:183-206.

Winer JA, Wenstrup JJ, Larue DT (1992) Patterns of GABAergic immunoreactivity define subdivisions of the mustached bat's medial geniculate body. J Comp Neurol 319:172-190.

Zook JM, Casseday JH (1982) Cytoarchitecture of auditory system in lower brainstem of the mustache bat, Pteronotus parnellii. J Comp Neurol 207:1-13.

Zook JM, Winer JA, Pollak GD, Bodenhamer RD (1985) Topology of the central nucleus of the mustache bat's inferior colliculus: correlation of single unit response properties and neuronal architecture. $\mathbf{J}$ Comp Neurol 231:530-546. 